\title{
Substrate degradation in high-Rayleigh-number reactive convection
}

\author{
T.J. Ward ${ }^{1}$, O.E Jensen ${ }^{2 a)}$, H. Power ${ }^{3}$, D.S. Riley ${ }^{1}$ \\ ${ }^{1}$ School of Mathematical Sciences, University of Nottingham, \\ U.K.; \\ ${ }^{2}$ School of Mathematics, University of Manchester, U.K.; \\ ${ }^{3}$ Faculty of Engineering, University of Nottingham, U.K.
}

(Dated: 26 October 2015)

We study buoyancy-induced convection of a solute in an ideal two-dimensional fluidsaturated porous medium, where the solute undergoes a second-order reaction with a chemical substrate that is fixed in the underlying matrix. Numerical simulations at high Rayleigh number show how a flow is established in which a thin dynamic boundary layer beneath the solute source feeds slender vertical plumes beneath. We examine how the substrate is reactively degraded, at a rate enhanced by convective mixing. For the case when the substrate is abundant, we derive a reduced-order model describing the slow degradation of the substrate, which is formulated as a novel onedimensional free-boundary problem. Numerical simulations and the reduced model reveal how, when the reaction is rapid compared to the convective timescale, the plumes propagate deep into the flow domain with reaction confined to a narrow region at their base. In contrast, slow reaction allows plumes to fill the domain before degradation of the substrate proceeds homogeneously. An alternative model with a thin reaction front captures the rapid degradation of the substrate when the solute concentration is relatively high.

a) Email address for correspondence: Oliver.Jensen@manchester.ac.uk 


\section{INTRODUCTION}

Convective transport in porous media is an important process with many engineering and industrial applications. ${ }^{1}$ One current focus lies in the sequestration of $\mathrm{CO}_{2}$, the long-term storage of $\mathrm{CO}_{2}$ in brine-saturated underground aquifers, motivating numerous recent studies into convective mixing. ${ }^{2}$ Upon injection, the buoyant $\mathrm{CO}_{2}$-rich fluid is initially trapped by a low-permeability cap-rock; mixing with the ambient brine facilitates more effective trapping mechanisms, such as dissolution of the $\mathrm{CO}_{2}$ and 'residual trapping' within the rock. ${ }^{2}$ Over geological timescales, $\mathrm{CO}_{2}$ may precipitate as carbonates due to a series of reactions ${ }^{3-5}$ with minerals in the host rock (known as 'mineral trapping') thus providing more stable storage. Natural analogues, where $\mathrm{CO}_{2}$ in solution has been in contact with the mineral formation

over geological periods, confirm that mineral trapping is a well-founded concept. ${ }^{6,7}$ The reaction timescales span many orders of magnitude, ${ }^{8,9}$ and although there is a lack of background data on the exact reaction rates, there is usually one controlling reaction during the mineralisation process that limits the production of precipitate. ${ }^{10}$ The long timescales associated with mineral trapping make conducting large-scale experiments difficult, emphasising a need for accurate numerical and theoretical studies of the problem.

Uncertainties in the physical and chemical structure of the host rock, however, make modelling the problem complicated. Studies of simplified systems, in which fundamental processes can be investigated in detail, provide an important foundation on which to build larger-scale simulations. The convective fingering arising in classical Rayleigh-Bénard porous-medium convection incorporates flow varying over different length-scales, which when coupled with reactions varying over widely disparate time-scales places great demands on computations attempting to integrate chemistry and flow; for a review of current literature on reactive flow see Emami-Meybodi et al. ${ }^{11}$ There is therefore significant interest in deriving low-order models that accurately capture the primary physical features of the flow.

Previous authors have studied the evolution of one-sided Rayleigh-Bénard porous-medium convection at large Rayleigh number $R a$ (where molecular diffusion is weak in comparison to convective flow - the flow regime applicable to $\mathrm{CO}_{2}$ sequestration ${ }^{12}$ ): a chemically inert solute introduced at the upper surface of the flow domain dissolves into the interstitial fluid, raising its density, generating convective mixing and resulting in solute being transported 
away from the source. Initially a diffusive boundary layer forms with a vertical concentration profile approximated by an error function. The diffusive layer subsequently loses stability and the system proceeds through successive convective regimes that have been described in detail by Slim ${ }^{1}$, Ennis-King and Paterson ${ }^{13}$, Riaz et al. ${ }^{14}$, Pau et al. ${ }^{15}$ and Backhaus et al. ${ }^{16}$ amongst others. Once convection is fully established, the flow is characterised by a thin dynamic boundary layer at the top of the flow domain, below which large, slender plumes penetrate downwards. The large plumes transport solute away from the boundary layer and create an upwelling of dilute solvent between the plumes. New plumes are created in the boundary layer and grow before being swept horizontally across the boundary layer, driven by the recirculation of the dilute solvent, towards the centre of the large plumes. As long as the plumes are shorter than the domain depth, the flux per unit length of solute into the domain, measured by a Sherwood number $S h$, remains approximately constant ${ }^{1,15,17}$ at $S h \approx 0.017 R a$. The solute transfer rate begins to fall after the plumes have penetrated to the base of the domain. ${ }^{1,18}$

In the presence of a reactive mineral substrate that is abundant relative to a dilute dissolved solute, the reaction can be described using first-order kinetics, at least over intermediate time scales. ${ }^{19-22}$ In this case the flow is parametrized by $R a$ and a Damköhler number $D a$ (a dimensionless reaction rate). The reaction creates a sink of solute in the domain and, at sufficiently large $D a$, limits the vertical penetration depth of the solute. At sufficiently large $R a$ the system establishes a statistically constant convective state in which the net flux of solute into the domain is balanced by the net loss of solute due to reaction, ${ }^{19}$ with the dynamic boundary layer regulating the flux of solute into the bulk. ${ }^{20}$ When $D a$ is sufficiently small, the domain saturates with solute and the reaction can be further approximated as zeroth-order, the problem being parametrized ${ }^{19,20}$ by $D a R a^{2}$. Alternatively, when the reaction is rapid in comparison to convective mixing, the vertical penetration depth of the solute is limited by the reaction; the first-order system is described by the depth-independent parameter ${ }^{19-22}$ $R a / D a$.

The asymptotic structure of the reactive flow for sufficiently large $R a$ resembles the structure of the non-reacting one-sided flow in many respects. A thin boundary layer forms near the upper boundary below which slender plumes penetrate deep into the domain. The timeaveraged solute flux follows the classical scaling, being proportional to $R a$, when convection 
has reached a statistically steady-state. However the flux also has a strong dependence on $D a$. At sufficiently large $D a$, simulations suggest that the flux is identical (at leading order) to that arising in classical one-sided Rayleigh-Bénard convection during intervals when the lower boundary has no influence, ${ }^{1}$ providing evidence that the flux in this regime is restricted by the boundary layer. ${ }^{20}$ At smaller $D a$, however, as solute fills the domain, the concentration outside the boundary layer rises and the flux across the boundary layer falls. Ward et al. ${ }^{20}$ showed how the solute flux is related to this concentration via a parameter that measures the excitability of the boundary layer (see (13) below).

Over longer timescales, reaction with the solute will deplete the amount of reactive mineral in the host matrix, expanding the flow field ${ }^{10}$ and effectively lowering $D a$ in regions that have had prolonged exposure to solute. Previous authors have modelled the reaction between dissolved solute and solid reactant as second-order. ${ }^{10,12}$ For large $D a$, reaction is limited to a thin region at the leading edge of the solute field. ${ }^{12}$ Studies by $\mathrm{Xu}$ et $a l .{ }^{23}$ and Zerai et $a l .{ }^{24}$ suggest that the porosity change caused by this reaction may be assumed negligible, as mineral volume change is too small to affect the dynamics of the system. In storage sites rich in calcite, for example, the dissolution of minerals in the solid matrix may be offset by the precipitation of silicate minerals, ${ }^{24}$ resulting in a net decrease of approximately $3 \%{ }^{23}$ in porosity.

Here we exploit an upscaling framework developed for first-order reaction ${ }^{20}$ to assess the effect of a second-order chemical reaction on the evolution of convective plumes as they penetrate into the domain; the model we use is described in Section II. In Section III we present numerical simulations for cases in which the substrate is depleted slowly or rapidly in comparison to convective mixing, and describe the effects of changing $D a$. We then compare these results to solutions of approximate low-order models derived in Section IV, yielding predictions of solute transfer rate, the rate of substrate depletion and the timeevolving penetration depth of the plumes. While the model we address has been simulated previously, ${ }^{10,12}$ we provide here a much more comprehensive survey of parameter space and identify key scaling properties of the flow. 


\section{MODEL}

We consider the dissolution of a solute, $\mathrm{C}$, in an ideal two-dimensional homogeneous porous medium (a solid matrix that is fully saturated with fluid) in which the solute undergoes a second-order reaction $\mathrm{C}+\mathrm{S} \rightarrow \mathrm{P}$, where species $\mathrm{S}$ is fixed in the host matrix and is depleted through its reaction with species $\mathrm{C}$, leaving the porosity, $\phi$, and the permeability, $K$, of the porous medium unchanged. Species C (but not the product $\mathrm{P}$ ) increases the solution density. Once species $\mathrm{S}$ is depleted from the interface between the fluid and porous matrix, the reaction terminates there and, as a result, the zone of reaction moves elsewhere.

We assume the rate at which species $\mathrm{S}$ is depleted is linearly proportional to $C^{*}$, the number of moles of species $\mathrm{C}$ per unit volume of saturating fluid, and to $S^{*}$, the number of moles of reacting mineral S per unit volume of the porous medium, as a fraction of $S_{I}^{*}$, the number of moles of reacting mineral initially present at the surface of the solid matrix per unit volume of the porous medium. The rate of change of species $\mathrm{S}$ results from the loss due to the surface reaction with species $\mathrm{C}$ via

$$
S_{t^{*}}^{*}=-\alpha \phi \frac{C^{*} S^{*}}{S_{I}^{*}},
$$

where $\alpha$ is a rate constant. The system is governed by (1), the convection-diffusion-reaction equation, the continuity equation and Darcy's law

$$
\begin{gathered}
\phi C_{t^{*}}^{*}+\nabla^{*} \cdot\left(\mathbf{u}^{*} C^{*}\right)=\phi D \nabla^{* 2} C^{*}-\alpha \phi \frac{C^{*} S^{*}}{S_{I}^{*}}, \\
\nabla^{*} \cdot \mathbf{u}^{*}=0, \quad \mathbf{u}^{*}=-\frac{K}{\mu}\left(\nabla^{*} p^{*}-\delta g C^{*} \hat{\mathbf{z}}\right),
\end{gathered}
$$

where $p^{*}, \mathbf{u}^{*} \equiv\left(u^{*}, w^{*}\right)$ and $\nabla^{*} \equiv\left(\partial / \partial x^{*}, \partial / \partial z^{*}\right)$ are the pressure, velocity and gradient operator respectively; gravity acts in the direction of increasing $z^{*}$. The parameters $\mu, g, D$ and $\delta$ are respectively the solvent viscosity, acceleration due to gravity, solute diffusivity and the density increase per unit concentration of solute; in the context of $\mathrm{CO}_{2}$ sequestration in a relatively high permeability storage site, such as the Utsira sand reservoir at Sleipner, ${ }^{25} \mu \approx$ $5 \times 10^{-4} \mathrm{~Pa} \mathrm{~s}, g \approx 10 \mathrm{~ms}^{-2}, D \approx 10^{-9} \mathrm{~m}^{2} / \mathrm{s}, \delta \approx 15 \mathrm{~kg} / \mathrm{m}^{3}, \phi \approx 0.3$ and $K \approx 5 \times 10^{-12} \mathrm{~m}^{2}$. We assume the solution density is linear in $C^{*}$ and that $\delta$ is sufficiently small for the Boussinesq approximation to apply. Reaction timescales during $\mathrm{CO}_{2}$ sequestration span many orders of magnitude and vary depending on the chemical composition of the storage site, as well 
as additional factors such as $\mathrm{pH}$, temperature and pressure ${ }^{8}$ we therefore do not place restrictions on potential values of $\alpha$ and $S_{I}^{*}$ but instead examine how their variation influences model predictions.

Equations $(1,2)$ are defined on a layer with horizontal coordinate $x^{*}$ and vertical depth $z^{*} \in\left[0, H^{*}\right]$; the domain length is taken to be infinite in the horizontal direction, and periodicity with period $L^{*}$ is assumed. Across $z^{*}=0$, a fixed concentration $\mathcal{C}^{*}$ of solute $\mathrm{C}$ is imposed for times $t^{*}>0$. Equations (2) are subject to the boundary conditions

$$
\begin{gathered}
C^{*}\left(x^{*}, 0, t^{*}\right)=\mathcal{C}^{*}, \quad C_{z^{*}}^{*}\left(x^{*}, H^{*}, t^{*}\right)=0, \\
w^{*}\left(x^{*}, 0, t^{*}\right)=0, \quad w^{*}\left(x^{*}, H^{*}, t^{*}\right)=0, \\
C^{*}\left(x^{*}, z^{*}, t^{*}\right)=C^{*}\left(x^{*}+L^{*}, z^{*}, t^{*}\right)=0, \quad u^{*}\left(x^{*}, z^{*}, t^{*}\right)=u^{*}\left(x^{*}+L^{*}, z^{*}, t^{*}\right) .
\end{gathered}
$$

Equations (1)-(3) are re-expressed using dimensionless variables $(x, z)=\left(x^{*}, z^{*}\right) / H^{*}$, $t=t^{*} K \delta g \mathcal{C}^{*} /\left(H^{*} \phi \mu\right), p=p^{*} /\left(\delta g H \mathcal{C}^{*}\right),\left(\mathbf{u}, \mathbf{u}_{I}\right)=\left(\mathbf{u}^{*}, \mathbf{u}_{I}^{*}\right) \mu /\left(K \delta g \mathcal{C}^{*}\right), C=C^{*} / \mathcal{C}^{*}$ and $S=S^{*} / S_{I}^{*}$ giving

$$
\begin{gathered}
\nabla \cdot \mathbf{u}=0, \quad \mathbf{u}=-\nabla p+C \hat{\mathbf{z}}, \\
C_{t}+\mathbf{u} \cdot \nabla C=R a^{-1} \nabla^{2} C-D a C S, \\
S_{t}=-D a \Omega C S,
\end{gathered}
$$

subject to the boundary conditions

$$
\begin{gathered}
C(x, 0, t)=1, \quad C_{z}(x, 1, t)=0, \\
w(x, 0, t)=0, \quad w(x, 1, t)=0, \\
C(x, z, t)=C(x+L, z, t)=0, \quad u(x, z, t)=u(x+L, z, t),
\end{gathered}
$$

where $L=L^{*} / H$ is the aspect ratio of the flow domain, and the dimensionless parameters are the Rayleigh, Damköhler and 'storage' numbers

$$
R a=\frac{H^{*} K \delta g \mathcal{C}^{*}}{\phi \mu D}, \quad D a=\frac{\alpha \phi H^{*} \mu S_{I}^{*}}{K \delta g \mathcal{C}^{*}}, \quad \Omega=\frac{\phi \mathcal{C}^{*}}{S_{I}^{*}} .
$$

$\Omega$ represents the abundance of dissolved solute relative to the concentration of reactive mineral initially present. At the Sleipner storage site, for example, initial reactions are likely to include calcite dissolution (this reacts relatively quickly, ${ }^{4}$ with $\alpha \approx 10^{-5} \mathrm{~s}^{-1}$ ) with 
$S_{I}^{*} \approx 0.067 \mathrm{~mol} / \mathrm{m}^{3}$ existing as shell fragments, ${ }^{26}$ giving $\Omega \approx 4$. However uncertainties in the physical and chemical structure of the storage site necessitate consideration of a wide range of parameter values: the limiting cases $\Omega \ll 1$ and $\Omega \gg 1$ discussed below represent the scenarios where the storage site is respectively rich or sparse in reactive mineral and provide bounds on possible outcomes. Writing $(4 a)$ in terms of the stream function $\psi$, defined as $(u, w)=\left(\psi_{z},-\psi_{x}\right)$, and eliminating the pressure, gives

$$
\psi_{z z}+\psi_{x x}=-C_{x}
$$

Equations $(4,5)$ depend on $R a, D a, \Omega$ and $L$; in this study $L$ is taken to be fixed and $R a \gg 1$.

We define the Sherwood number, $S h$, a dimensionless measure of the solute flux per unit length across the upper boundary, by

$$
S h=-\left.\frac{1}{L} \int_{0}^{L} C_{z}\right|_{z=0} \mathrm{~d} x
$$

with the corresponding dimensional flux per unit length being $\left(\phi D \mathcal{C}^{*} / H\right) S h$.

Integrating (4b), subject to (5), yields measures of the total number of moles of dissolved solute, $M_{C} \equiv \int_{0}^{1} \int_{0}^{L} C \mathrm{~d} x \mathrm{~d} z$, and of the total number of moles of surface reactant in the host rock, $M_{S} \equiv \int_{0}^{1} \int_{0}^{L} S \mathrm{~d} x \mathrm{~d} z$, which satisfy

$$
M_{C t}=(L S h / R a)-D a \int_{0}^{1} \int_{0}^{L} C S \mathrm{~d} x \mathrm{~d} z, \quad M_{S t}=-D a \Omega \int_{0}^{1} \int_{0}^{L} C S \mathrm{~d} x \mathrm{~d} z,
$$

so that

$$
M_{C t}-\Omega^{-1} M_{S t}=L R a^{-1} S h .
$$

Simulations reported below are summarised using a set of representative variables, defined as follows. We use a horizontal average $\langle\cdot\rangle$ to describe vertical solute distributions. The plume penetration depth $z_{0}$ is estimated numerically as the minimum value $z$ for which $\langle C\rangle \leq 0.01$; when $\left.\langle C\rangle\right|_{z=1}$ is nonzero we take $z_{0}=1$. We estimate the horizontal wavenumber of convective plumes by defining $\tilde{C}=C-\langle C\rangle$ and computing $^{1}$

$$
k=\left.\sqrt{\left\langle\tilde{C}_{x}^{2}\right\rangle /\left\langle\tilde{C}^{2}\right\rangle}\right|_{z=z_{0} / 2} .
$$

Close to the boundary near $z=0,\langle C\rangle$ decreases monotonically with $z$ before reaching a turning point. The depth of the boundary layer is estimated numerically as $z_{\lambda}=\min _{z}\left\{\langle C\rangle_{z z}=0\right\}$ 
and the corresponding concentration of $C$ at the edge of the boundary layer is determined as $\lambda=\left.\langle C\rangle\right|_{z=z_{\lambda}}$. A measure of the number of moles per unit width of depleted reacting mineral is defined as

$$
\mathcal{M} \equiv \int_{0}^{1}(1-\langle S\rangle) \mathrm{d} z .
$$

For later reference, we note that for $R a \gg 1, S h$ is well approximated ${ }^{20}$ by

$$
S h \approx J_{0} R a, \quad J_{0}=\frac{2(1-\lambda)^{2}}{\pi \Delta_{c}}
$$

when $\Omega=0$ and the convection is in a statistically steady state. Here $\Delta_{c}$ is a parameter (with value in the range 15-20) characterising the excitability of the dynamic sub-surface boundary layer.

\section{A. Numerical method}

Equations $(4 b, 4 c, 5,7)$ were solved using a spectral method, ${ }^{27}$ approximating $C, \psi$ and $S$ by $N$ Chebyshev polynomials of the first kind (satisfying the vertical boundary conditions) in the $z$-direction and evaluating the governing equations at the Chebyshev collocation points $k_{j}=\cos (2 j-1) \pi /(2 N)$ for $J=1, \ldots, N$. Fourier series, with $M$ modes, were used to approximate the solutions in the horizontal direction, enforcing periodic boundary conditions along the vertical boundaries. Solutions are assumed to be of the form

$$
\begin{gathered}
C(x, z)=\sum_{m=-M / 2}^{M / 2} C_{m}(z) \exp (2 i m \pi x / L), \quad \psi(x, z)=\sum_{m=-M / 2}^{M / 2} \psi_{m}(z) \exp (2 i m \pi x / L) \\
S(x, z)=\sum_{m=-M / 2}^{M / 2} S_{m}(z) \exp (2 i m \pi x / L) .
\end{gathered}
$$

Solving $(4 b, 4 c, 5,7)$ in Fourier space decouples the system into $M$ linear equations, each of order $N$, which were solved in time using a leapfrog scheme, ${ }^{20}$ this requires solutions at time steps $n-1\left(C^{n-1}, \psi^{n-1}, S^{n-1}\right)$ and $n,\left(C^{n}, \psi^{n}, S^{n}\right)$ in order to evaluate solutions at step $n+1$. The advection term in $(4 b)$ and the nonlinear reaction term in $(4 b, c)$ were evaluated at time step $n$ and transformed into Fourier space to give the nonlinear terms Conv $^{n}$ and React $^{n}$ respectively. The $m t h$ components of $(4 b, c)$ were then solved using

$$
\frac{C_{m}^{n+1}-C_{m}^{n-1}}{\delta t / 2}+2 \operatorname{Conv}_{m}^{n}=R a^{-1}\left(\mathrm{D}_{\mathrm{zz}}-(2 m \pi / L)^{2} \mathrm{I}\right)\left(C_{m}^{n+1}+C_{m}^{n-1}\right)-2 \text { Da React }{ }_{m}^{n},
$$




$$
\frac{S_{m}^{n+1}-S_{m}^{n-1}}{\delta t / 2}=-2 D a \Omega \text { React }_{m}^{n},
$$

to find $\left(C_{m}^{n+1}, \psi_{m}^{n+1}, S_{m}^{n+1}\right)$, where $\mathbf{D}_{\mathrm{zz}}, \mathrm{I}$, and $\delta t$ are the second-order differential operator acting on the rescaled Chebyshev polynomials, the identity matrix and the time step, respectively. Having solved (15) for $m=-M / 2 \ldots, M / 2$, the $m$ th component of the stream function was obtained using

$$
\left(\mathrm{D}_{\mathrm{zz}}-(2 m \pi / L)^{2} \mathrm{I}\right) \psi_{m}^{n+1}=-(2 i m \pi / L) C_{m}^{n+1}
$$

For all simulations we used the initial condition $C(x, z, 0)=\cosh (700(1-z)) / \cosh (700)$, $S(x, z, 0)=1$, ensuring $C$ initially decays quickly and smoothly over $z$; the forward Euler method was used to obtain the second initial condition for the leapfrog scheme. We also applied the Robert-Asselin filter to (15) to suppress any computational modes resulting in time-splitting instability. ${ }^{28}$ Solutions to $(4 b, 4 c, 5,7)$ with $\Omega=0$ for horizontally-averaged concentration profiles and $S h$ (using (8)) were compared with previously published results. ${ }^{20}$ Equations $(9,10)$ were also used as computational checks on the numerical scheme. The numbers of Chebyshev and Fourier modes were increased, and the time-stepping interval decreased, until the measured quantities where statistically equivalent.

\section{NUMERICAL SIMULATIONS}

All the simulations presented below were conducted with $R a=5000$ and $L=\pi$. We provide detailed illustrations of the flow at small and large $\Omega$ and then describe the effects of changing $D a$ and $\Omega$ independently. Simulations will be compared to approximations from simplified models in Section IV below.

\section{A. Slow substrate degradation: $D a=1, \Omega=0.01$}

Small $\Omega$ represents the case in which species $S$ is initially abundant relative to the solute, implying that the substrate concentration falls relatively slowly as the solute decays.

Simulations of $(4,5)$ for $D a=1, \Omega=0.01$ reveal a diffusive layer that forms and quickly loses stability to convective fingering at $t \approx 0.7$. Fully established convection proceeds, as illustrated in Figures 1-3, which then evolves slowly as the reacting mineral is depleted. At 
$t \approx 2.5$ a very thin dynamic boundary layer forms near the top of the domain, across which $\langle C\rangle$ falls to approximately $30 \%$ of its value along the top of the domain (Figure $1(a-c)$ ), and beneath which slender vertical plumes are established. As demonstrated previously, ${ }^{20}$ as long as $S \approx 1$ the boundary layer has thickness of order $1 / R a$, the plumes beneath have depth of order $1 / D a$ and the plume wavenumber scales like $R a^{1 / 2}$. As time passes the mineral begins to deplete (Figure 3), initially without substantial elongation of the plumes. The flow field fluctuates but is initially most vigorous immediately beneath the boundary layer (Figure 2). Because convective mixing is rapid compared to the rate of substrate degradation, the inherent disorder in the convective plumes suppresses horizontal $x$-variations in the $S$ field. However the $x$-variation in $S$ becomes more pronounced at later times (Figure $3(d)$ ).

Figure 4 shows horizontally-averaged profiles of $C, S$, the root-mean-squared perturbations $\tilde{C}(\equiv C-\langle C\rangle), \tilde{S}(\equiv S-\langle S\rangle)$ and the stream function plotted against $z / z_{0}(t)$, where $z_{0}(t)$ is the plume penetration depth (shown as a function of time in Figure 5(a) below), during the phase in which the plumes are elongating; the dotted curves show for comparison the solutions to a simplified model derived in Section IV A below. Outside the boundary layer near $z=0,\langle S\rangle$ decreases monotonically in $t$ when plotted against rescaled depth. In contrast, the shape of $\langle C\rangle$ changes at a slower rate, increasing slightly from an initially linear profile. Eventually, $S$ is completely depleted near $z=0$ and the distribution of reacting mineral exhibits a travelling-wave structure that propagates downwards towards the base of the domain (Figure 3). $\langle S\rangle$ has a sigmoidal shape that narrows with respect to $z / z_{0}$ (Figure $4(b))$ but which has approximately uniform width with respect to the $z$-coordinate (Figure $3(c-e))$. There is a corresponding change in the shape of the mean flow field (Figure 2), with the strongest flow moving away from the upper boundary into the interior of the domain. Horizontal perturbations in $C$, measured by $\left\langle\tilde{C}^{2}\right\rangle^{1 / 2}$, are of comparable magnitude to the horizontally averaged field (Figures 4a,d) whereas perturbations in the $S$ field are significantly weaker than $\langle S\rangle$, at least at early times (Figures $4 \mathrm{~b}, \mathrm{e}$ ); this is a result of lateral fluctuations of the plumes reducing horizontal gradients of the $S$ field.

Macroscopic features of the time-evolving flow field for $\Omega=0.01$ are illustrated in Figure 5. After an initial transient, the flow undergoes an extended slowly-varying phase during which the substrate is slowly eroded and the plumes descend slowly towards the base of the domain, reaching it at $t \approx 3500$ (indicated by the cross marked I in the figure); thereafter the flow 

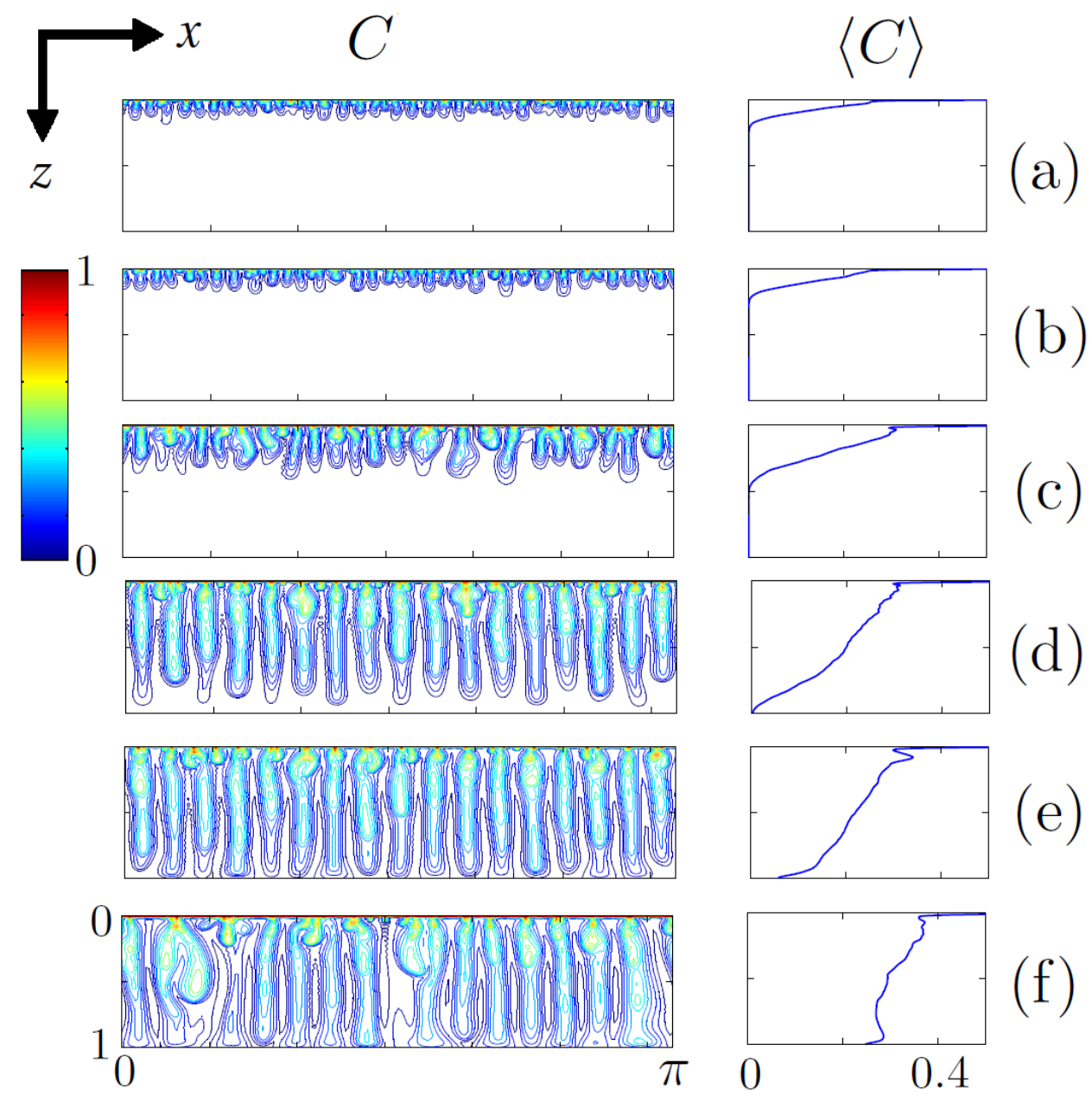

(f)

FIG. 1. Snapshots of the $C$ field for $R a=5000, D a=1, \Omega=0.01$ and $L=\pi$, satisfying $(4,5)$, and taken at $(a) t=10,(b) 100,(c)$ 1000, $(d) 3500(e) 4250$ and $(f)$ 5000. Contours (left) show lines along which $C$ is constant; regions of uniform concentration are blank. The right-hand panels show the vertical structure of the horizontally-averaged $C$ field.

shuts down relatively abruptly. The long intermediate phase is illustrated by tracking the location of the base of the plumes $z_{0}(t) ; z_{0}$ increases approximately linearly in time until it reaches unity. The Sherwood number shows only minor variations during the intermediate phase (Figure $5(b))$. The dashed line in Figure $5(b)$ shows the approximation $S h=J_{0} R a$ (see (13)) using $J_{0}=0.02$, marginally above the anticipated flux ${ }^{20} J_{0}=0.017$ predicted in 


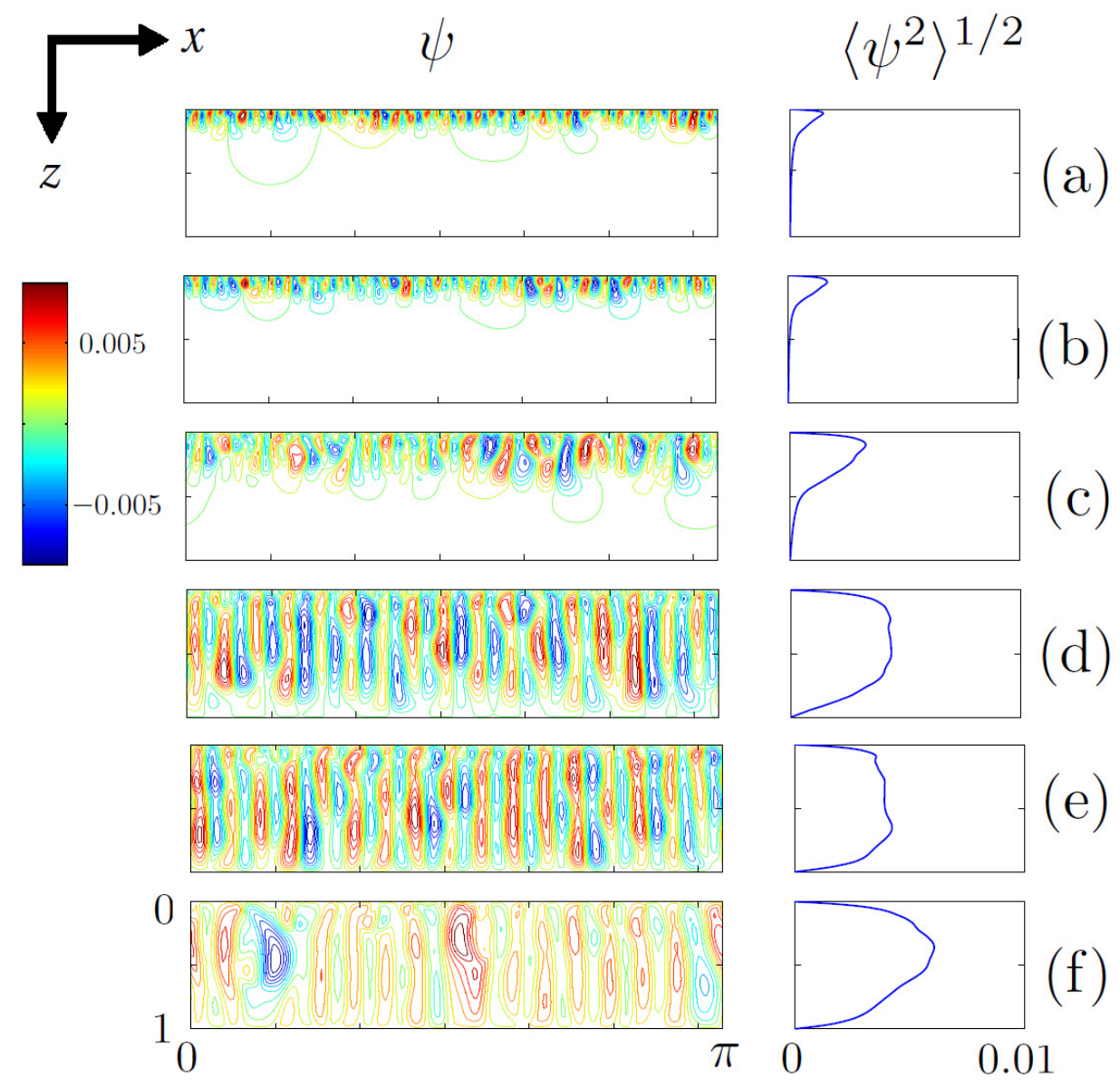

FIG. 2. Snapshots of the stream-function for parameters and times corresponding to Figure 1. Contours (left) show streamlines. The right-hand panels show the vertical structure of the horizontallyaveraged $\psi$ field.

the limit $R a \rightarrow \infty$ for $S=1$ (and also reported for high- $R a$ one-sided convection in the absence of chemical reaction $\left.{ }^{1,15}\right)$. The simulations lie sufficiently close to this asymptotic threshold, and appear sufficiently insensitive to the local depletion of the $S$ field, to suggest that the solute transfer into the domain is regulated primarily by the dynamic boundary layer near $z=0$, at least while the bottom of the domain has no influence on the overall flow. Figure $5(c)$ shows that the solute concentration immediately beneath the boundary layer, represented by $\lambda$, also remains approximately constant over the intermediate phase; it is well 

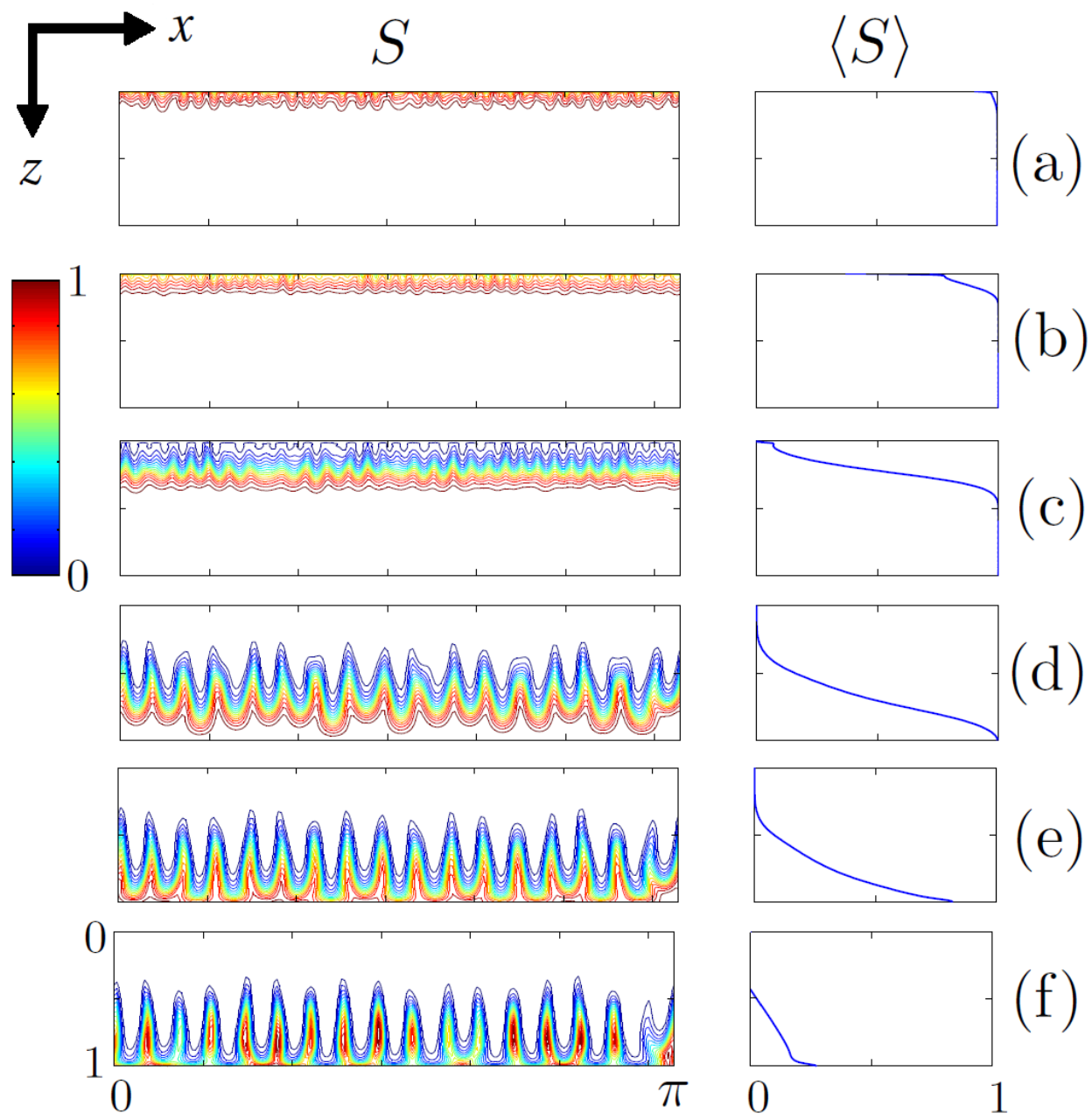

FIG. 3. Snapshots of the $S$ field for parameters and times corresponding to Figure 1. Contours (left) show lines along which $S$ is constant; regions of uniform concentration $(S=0$ above contours in (d-f), $S=1$ below contours in (a-d)) are blank. The right-hand panels show the vertical structure of the horizontally-averaged $S$ field.

approximated by $\lambda=0.29$, close to the value reported previously. ${ }^{20}$ The average horizontal plume wavenumber at $z=z_{0} / 2$ (Figure $5(c)$ ) is initially constant but for sufficiently large $t$ it appears to decay like $t^{-1 / 2}$ (Figure $5(d)$ ), before falling rapidly as the flow shuts down.

Figures $5(e)$ and $(f)$ respectively show measures of the amount of unreacted $C$ in the domain, $M_{C}$, and the amount that $S$ has been depleted, $\mathcal{M}$. During the intermediate phase, 


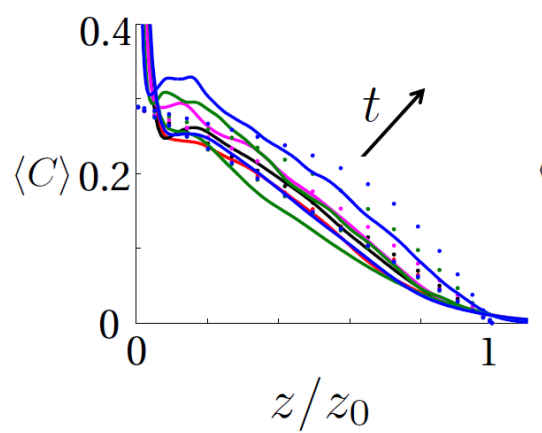

(a)

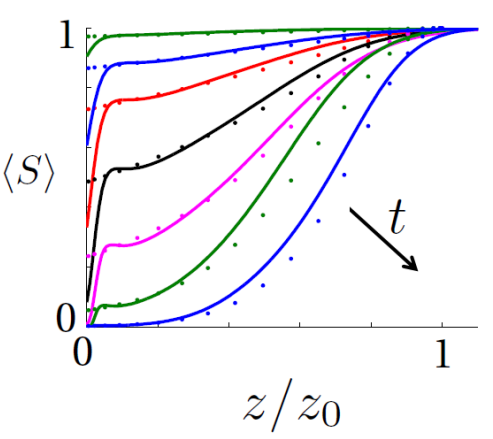

(b)

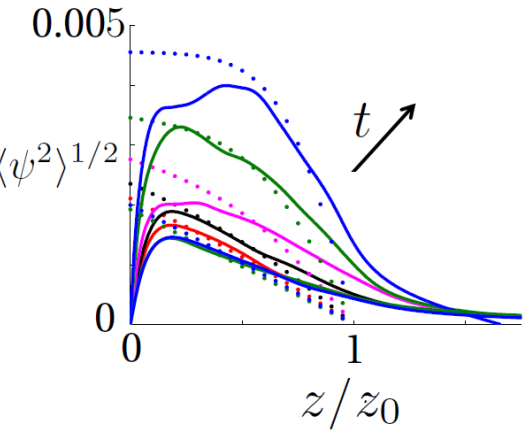

(c)

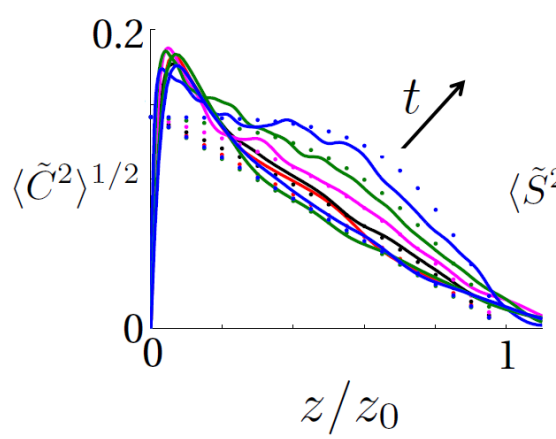

(d)

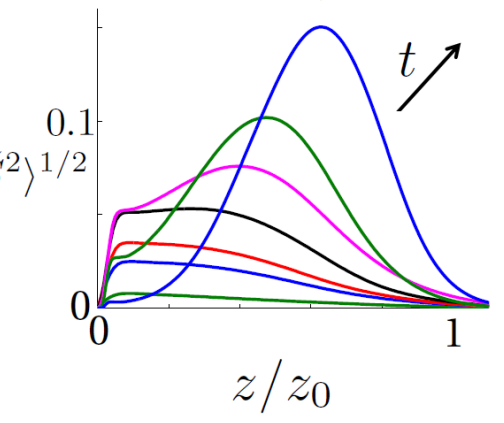

(e)

FIG. 4. Solid curves show the horizontally-averaged $C$ field (a) and $S$ field (b) plotted against $z / z_{0}$ calculated from $(4,5)$ using $R a=5000, D a=1, \Omega=0.01$ and $L=\pi$ and plotted at $t=10,50,100,250,500,1000$ and 2000. Dots show solutions to the small- $\Omega$ model (32) for $\lambda=0.29, J_{0}=0.02$ at corresponding times. The horizontally-averaged root-mean-square of (c) the stream function, (d) the variation in the $C$ field and (e) the variation in the $S$ field are shown at corresponding times, comparing simulations (solid) to the reduced model (dots).

for which the flux of $C$ into the domain remains approximately constant, $M_{C}$ and $\mathcal{M}$ increase accordingly at a roughly constant rate. Once the plumes touch the bottom of the domain (point I in Figure 5), the system starts to shut down, with $S h$ falling and $\lambda$ rising. At the start of the shut-down phase, $S$ is totally depleted near the top of the domain, leaving only small regions of unreacted substrate at the bottom of the domain between the plumes (Figure $3(f)$ ). Thereafter the amount of remaining $S$ decreases until it is fully depleted at $t \approx 5500$ (the crosses labelled II in Figure 5 indicate the minimum time at which $\mathcal{M}>0.99$ ). 


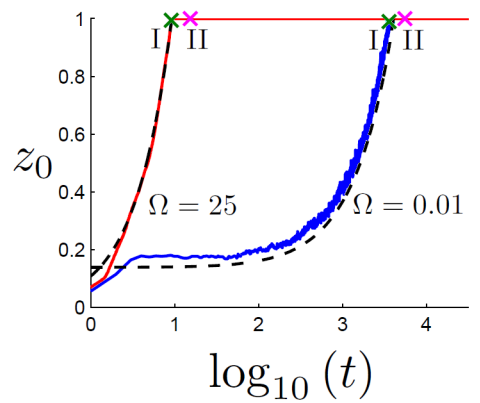

(a)

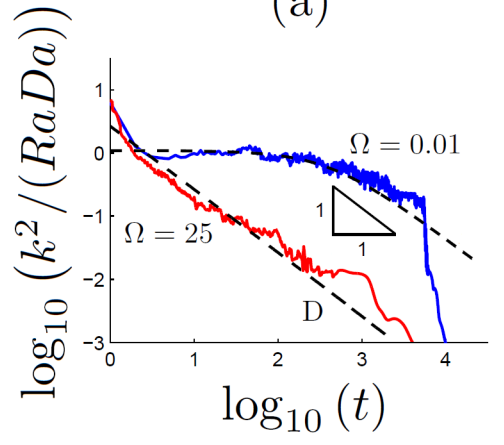

(d)

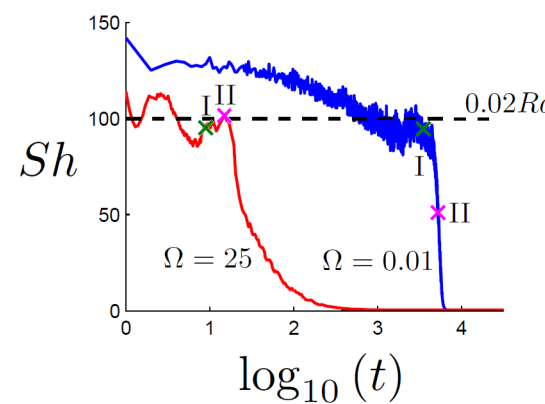

(b)

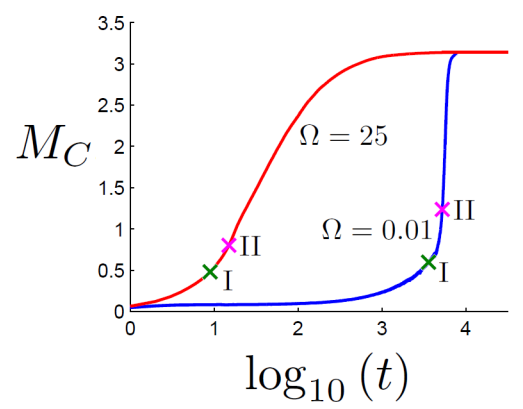

(e)

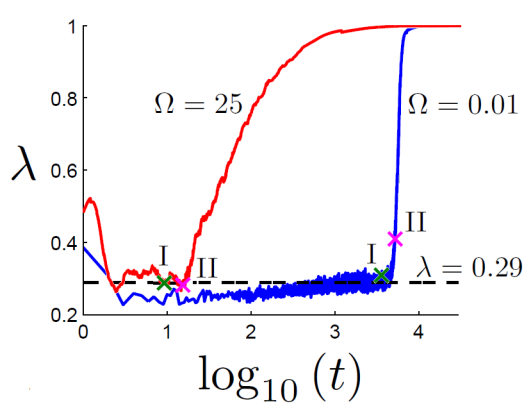

(c)

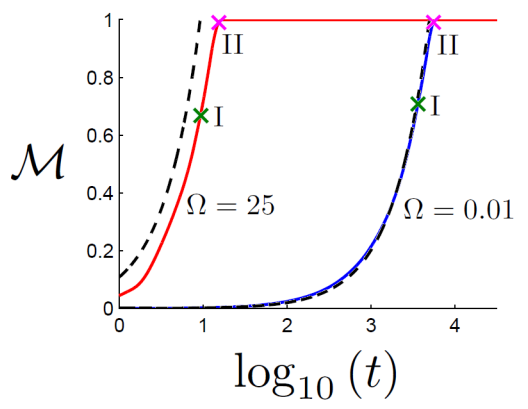

(f)

FIG. 5. Numerical results for $R a=5000, D a=1, L=\pi$ and $\Omega=0.01$ (blue) and $\Omega=25$ (red) calculated from $(4,5)$. (a) shows the plume penetration depth $z_{0}$; dashed lines indicate predictions from the reduced models (32) for $\Omega=0.01$ and (48) for $\Omega=25$. (b) and (c) respectively show the Sherwood number, $S h$, and the value of $C$ at the outer edge of the boundary layer, $\lambda$. Dashed lines show the approximations $J_{0}=0.02 R a$ and $\lambda=0.29$ respectively used in the reduced models. $(d)$ shows the mid-plume wavenumber $k /(R a D a)^{1 / 2}$, calculated using $(4,5,11)$ (solid curve); dashed curves illustrate the wavenumber calculated from the small- $\Omega$ and large- $\Omega$ models (32) and (48) and the triangle indicates decay of $k$ like $t^{-1 / 2}$. $(e)$ and $(f)$ show the quantity of unreacted $C, M_{C}$ and unreacted $S, \mathcal{M}$ in the domain. Crosses, labelled I and II, signify the approximate times at which tips of the plumes first encounter the bottom of the domain and $\mathcal{M}>0.99$ respectively.

The final depletion of $S$ has little effect on $S h$ and $M_{C}$ (Figure 5), as the convection weakens and the domain becomes saturated with solute. 


\section{B. Rapid substrate degradation: $D a=1, \Omega=25$}

Large $\Omega$ represents the case in which the species $S$ is initially present at low concentrations relative to the solute, implying that the substrate can be depleted rapidly once solute is added to the domain. Equation $(4 c)$ demonstrates that, when $\Omega$ is large, the substrate degrades quickly in regions where both $S$ and $C$ are present. The rapid reaction promotes the formation of sharp interfaces separating regions where $C>0$ and $S=0$ from those in which $C=0$ and $S=1$. We illustrate this with simulations of $(4,5)$ for $D a=1$ and $\Omega=25$ shown in Figures 6, 7; the evolution of the macroscopic features of the flow field is illustrated in Figure 5.

As in the small- $\Omega$ example, the flow has an initial transient phase during which a diffusive boundary layer loses stability to convective fingering (Figure 6(a)). A thin dynamic boundary layer then forms at the top of the domain, characterised by an approximate $70 \%$ fall in $\langle C\rangle$, beneath which slender plumes grow (Figure 6(b)). The penetration depth of the plumes increases linearly with time (Figure $5(a)$ ), significantly more rapidly than in the previous example; $S h$ and $\lambda$ again remain approximately constant at values similar to the $\Omega=0.01$ case (Figures 5(b,c) respectively), at least until the plumes reach the base of the domain $(t \approx 9.3$, point $\mathrm{I})$. The average horizontal wavenumber at $z=z_{0} / 2$ again decays like $t^{-1 / 2}$ (Figure 5(d)).

The substrate is rapidly depleted where the plumes descend, leaving areas between the plumes where $S$ is unreacted (Figure $7(b, c)$ ). Once the plumes reach the bottom of the domain, solute spreads horizontally across the lower boundary (Figure $6(d)$ ), depleting $S$ and leaving isolated pockets of unreacted $S$ (Figure $7(e, f)$ ). Thereafter, as saturated solvent recirculates to the top boundary layer, $S h$ falls, $\lambda$ rises and there is an increase in the rate of change of $M_{C}$ (Figure 5). As the domain saturates with solute, the isolated pockets of $S$ decrease in size (Figure $7(e)$ ) until $S$ is totally depleted at $t \approx 15.4$ (point II in Figure 5 ). Once chemically inert, the system shuts down at a rate comparable to the previous example (Figures $5(e, f))$.

Figure 8 shows the evolution of horizontally-averaged profiles of $C, S$, the root-mean-

squared $\tilde{C}, \tilde{S}$ and the stream function; dotted curves show for comparison solutions to the reduced model derived in Section IV C below. In the bulk of the domain $\langle C\rangle$ decreases 

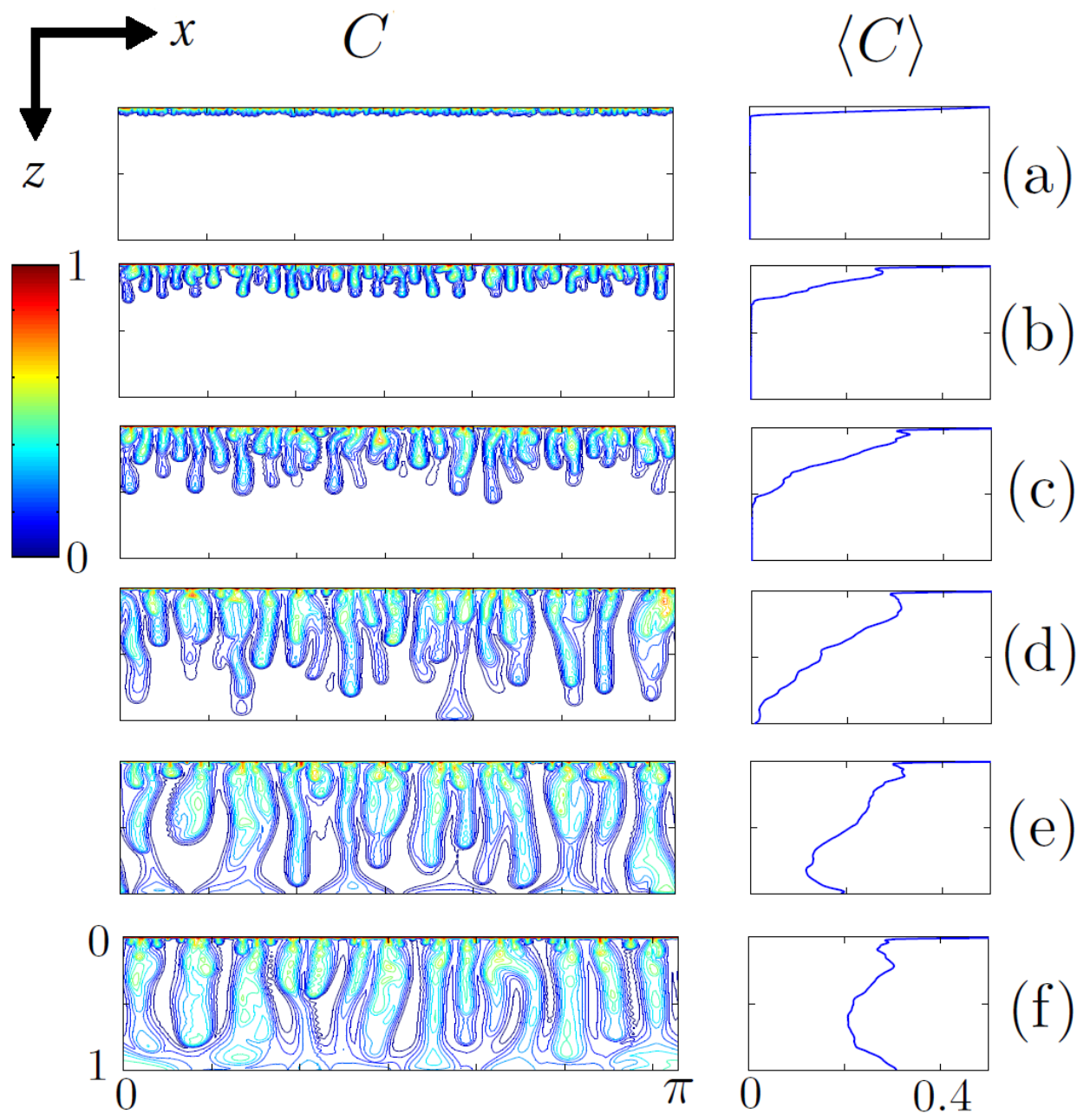

FIG. 6. Snapshots of the $C$ field for $R a=5000, D a=1, \Omega=25$ and $L=\pi$, satisfying $(4,5)$, and taken at $(a) t=1,(b) 2.5,(c) 5,(d) 9.3,(e) 12.5$ and $(f)$ 15. Contours (left) show lines along which $C$ is constant; regions of uniform concentration are blank. The right-hand panels show the vertical structure of the horizontally-averaged $C$ field.

linearly with respect to $z$. Although the reaction zone is thin, the distribution of $S$ is patchy at later times (Figure 7), increasing the vertical dispersion of the $\langle S\rangle$ field. The stream function increases in magnitude over later times with the most vigorous flow moving towards the centre of the domain. In contrast to the example shown in Figures 1-4, for large $\Omega$ the plumes erode pronounced channels in the $S$ field which appear to suppress 

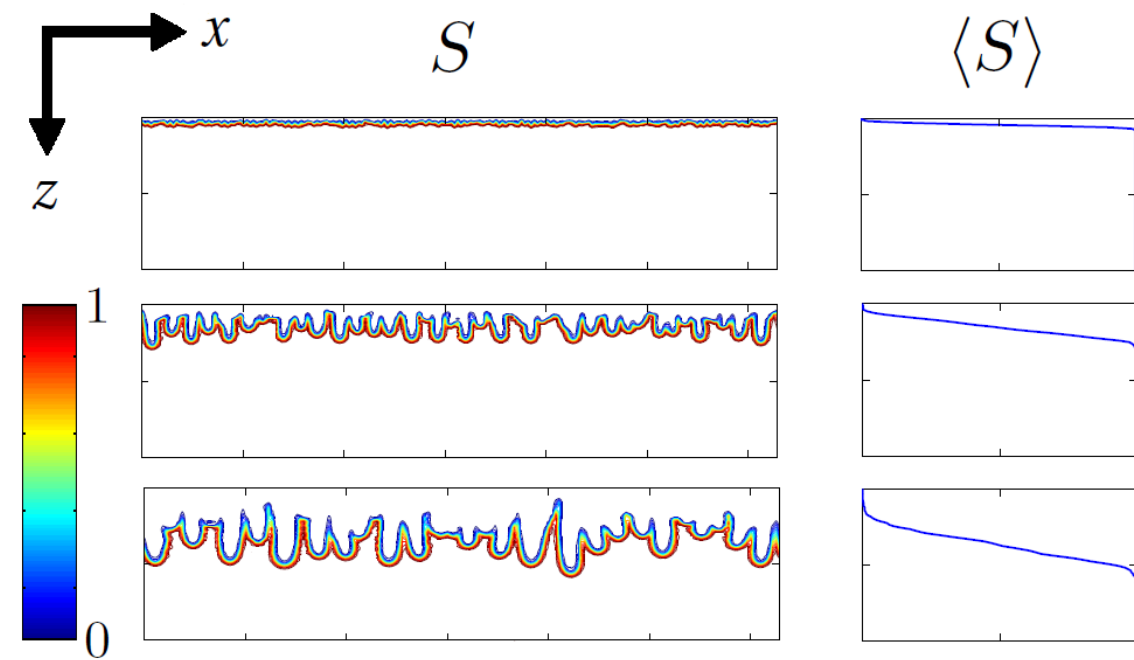

(a)

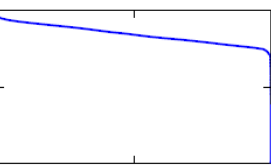

(b)

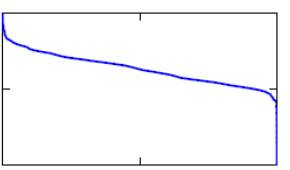

(c)
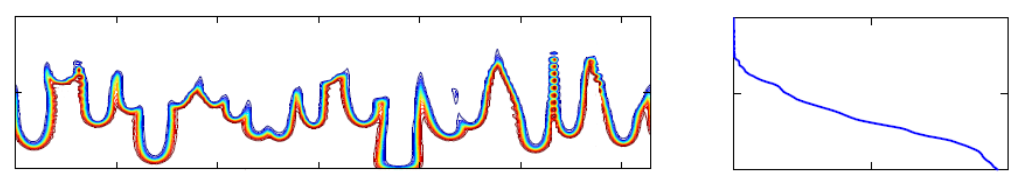

(d)
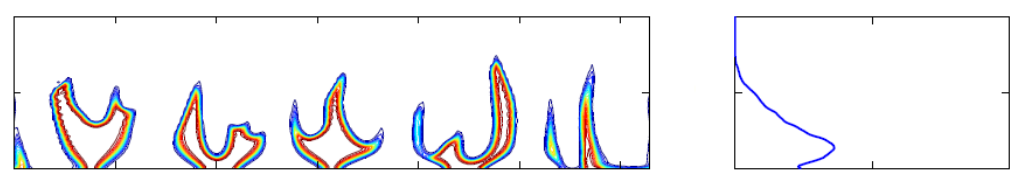

(e)
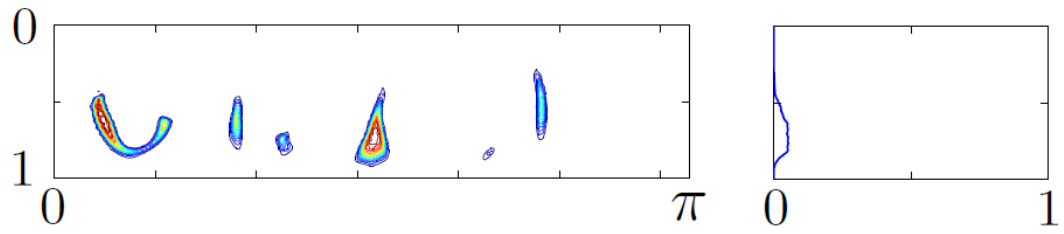

(f)

FIG. 7. Snapshots of the $S$ field for parameter values and times corresponding to Figure 6. Contours (left) show lines along which $S$ is constant; regions of uniform concentration are blank. The righthand panels show the vertical structure of the horizontally-averaged $S$ field.

lateral fluctuations of the plumes, leaving both $\tilde{C}$ and $\tilde{S}$ comparable in magnitude to the horizontally averaged fields (see Figures $8(\mathrm{a}, \mathrm{d})$ and (b,e) respectively). 


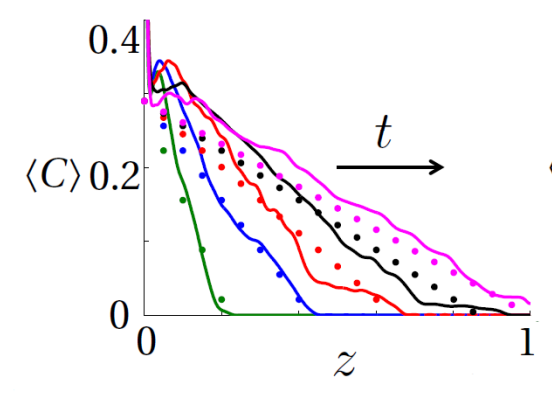

(a)

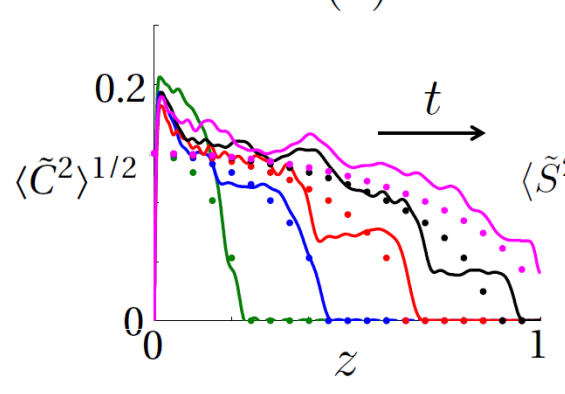

(d)

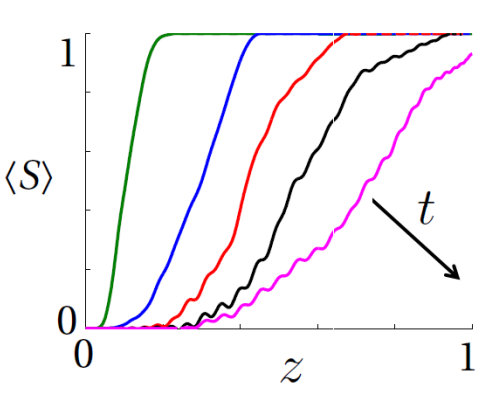

(b)

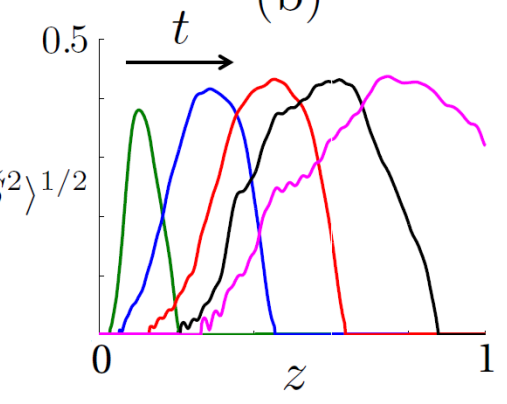

(e)

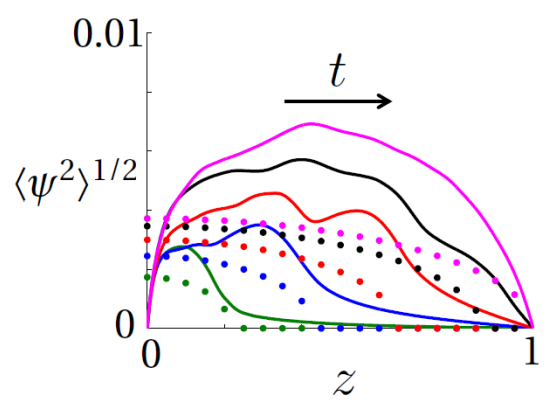

(c)

FIG. 8. Plots of the horizontally-averaged $C$ field (a) and $S$ field (b) against $z$ calculated from $(4,5)$ using $R a=5000, D a=1, \Omega=25$ and $L=\pi$ and plotted at $t=2,4,6,8$ and 10 . The horizontally-averaged root-mean-square of (c) the stream function, (d) the variation in the $C$ field and (e) the variation in the $S$ field are shown at corresponding times. Dotted curves show solutions to the large- $\Omega$ reduced model $(45,46,48)$ using $\lambda=0.29, J_{0}=0.02$ at corresponding times.

\section{The effect of changing $D a$}

Simulations for different values of $D a$ provide insight into the dependence of the flow structure on reaction rate. We return to the case in which the substrate is abundant relative to the solute $(\Omega=0.01)$ but now consider variation in the reaction rate relative to the convective timescale. We first demonstrate that the dynamic boundary layer near $z=0$ continues to regulate the flux of solute into the domain.

Figure $9(a)$ shows the measure of the number of moles per unit length of depleted substrate $\mathcal{M}$, defined in (12), plotted against the time scale $\tau=D a \Omega t$. The data for $D a \mathcal{M}$ in Figure $9(a)$ are well approximated by the dashed straight line with gradient $J_{0}=0.02$, 


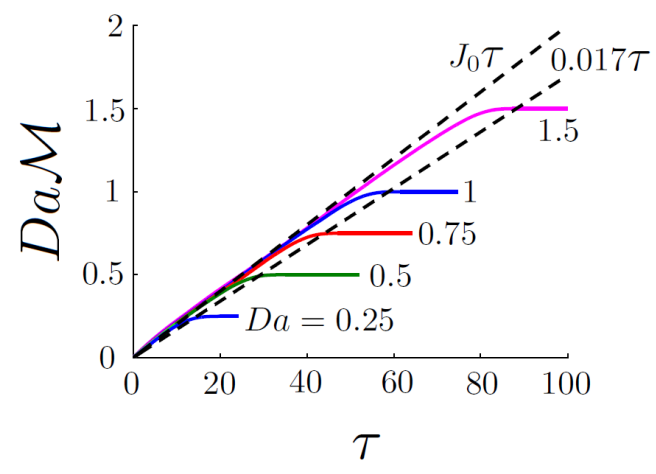

(a)

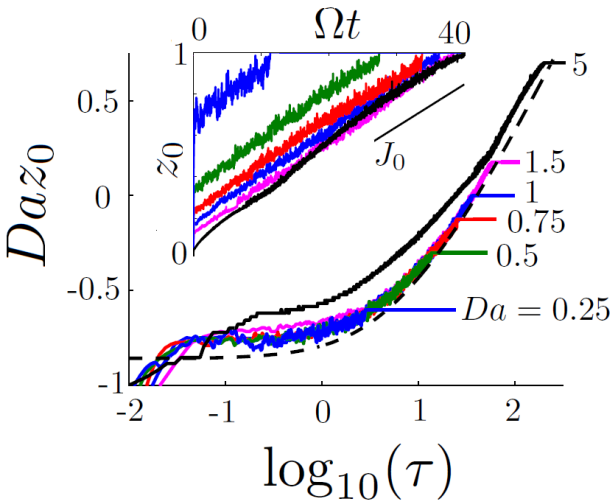

(b)

FIG. 9. (a) The measure of the number of moles of depleted substrate reactant $\mathcal{M}$, and (b) plume penetration depth $z_{0}$ plotted against $\tau=D a \Omega t$, using solutions to $(4,5)$ for $R a=5000, \Omega=$ $0.01, L=\pi$ and $D a=0.25,0.5,0.75,1$ and 1.5. The dashed line in (b) shows the solution to (32) using $J_{0}=0.02$ and $\lambda=0.29$.

indicating steady flux of solute across $z=0$; as explained in Section IV A below, this is consistent with $S h \approx 0.02 R a=100$ in Figure $5(b)$. This value of $J_{0}$ lies slightly above the $R a \rightarrow \infty$ asymptote of $0.017 R a$ (Figure $5(b)$ ), reflecting the fact that the simulation is conducted at finite $R a$.

Until the plume hits the base of the domain, the plume penetration depth $z_{0}$ also increases approximately linearly with time, as illustrated in the inset to Figure $9(b)$. At large times, and for larger $D a, z_{0}$ increases independently of $D a$ at a rate of $J_{0} \Omega$ with respect to $t$, whereas for smaller $D a$, the data for $z_{0}$ collapse when $D a z_{0}$ is plotted against $\tau=D a \Omega t$ for different $D a$, for reasons explained in Section IV A below. Figure 5(b) illustrates that the primary effect of increasing $D a$ is to increase the effective depth of the domain relative to the lengthscale of the growing plumes.

When $D a \ll 1$ and $\Omega \ll 1$, the reaction is sufficiently slow for the plumes initially to fill the domain in a manner reminiscent of one-sided convection, before eventually the solute concentration equilibrates across the domain; thereafter it elevates slowly as the substrate is depleted. Figure 10 shows horizontally-averaged profiles of $C$ and $S$ during this final phase 


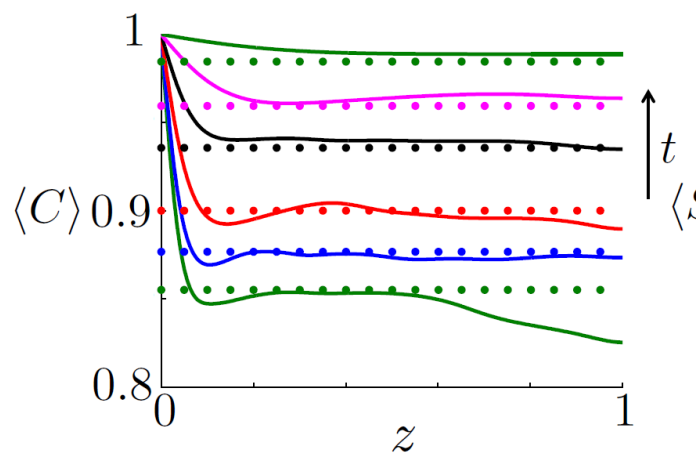

(a)

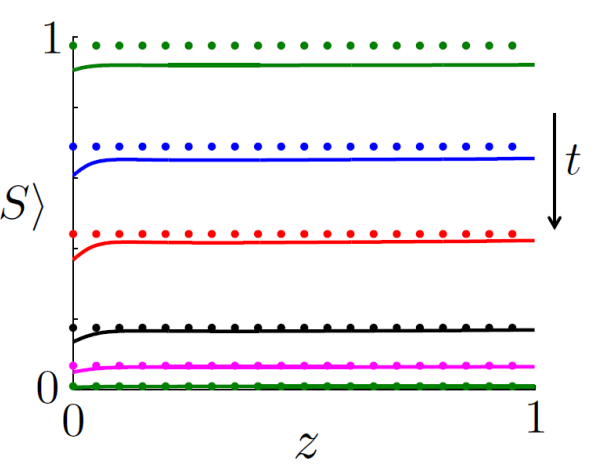

(b)

FIG. 10. Solid curves show the horizontally-averaged $C$ field (a) and $S$ field (b) calculated from $(4,5)$ using $R a=5000, D a=0.001, \Omega=0.01$ and $L=\pi$ and plotted at $t=1 \times 10^{4}, 5 \times 10^{4}, 1 \times$ $10^{5}, 2 \times 10^{5}, 3 \times 10^{5}$ and $5 \times 10^{5}$. Dots show solutions to the small- $D a$ model equations $(38,39)$ and $\bar{C}=\lambda$ for $\Delta_{c}=16$ and $\tau_{0}=0.07$ at corresponding times.

for $D a=0.001$ and $\Omega=0.01$; the dotted curves show for comparison the solutions to the simplified model derived in Section IV B below. Figure 10 shows that $\langle C\rangle$ and $\langle S\rangle$ are almost uniform in the bulk of the domain while evolving slowly with respect to $t$.

To illustrate the dynamics of this slow evolution, the macroscopic features of the timeevolving flow for $D a=0.01, \Omega=0.01$ and $D a=0.001, \Omega=0.01$ are illustrated in Figure 11 . During the initial box-filling phase in both cases (prior to the point marked I in Figure 11), $\lambda \approx 0.3$ and $S h \approx 0.02 R a$; the lower boundary then has an influence, driving a transition to values in which the system is in a quasi-steady balance in which $\lambda$ is equivalent to the case when $\Omega=0$ and $S=1$. At this stage, $S h, M_{C}$, and $\lambda$ plateau at respective values close to $28,1.78$ and 0.6 for $D a=0.01$ and $4.23,2.66$ and 0.83 for $D a=0.001$, approximately equal to the values obtained for $\Omega=0$ (red curve in Figures 11(a,b,d)); for smaller $D a$ the domain is more saturated with solute in the balanced state. Finally, over very long timescales, the substrate is depleted, causing $\lambda, \mathcal{M}$ and $M_{C}$ to rise and $S h$ to fall as the convection slowly shuts down. Decreasing $D a$ increases the timescales for the substrate to become fully depleted (labelled by II in figure 11) and for the domain to fully saturate with $C$ (Figures $11(c, d))$. In contrast to Figure 5 (for $D a=O(1)$ ), Figure 11 shows (for $D a \ll 1)$ 


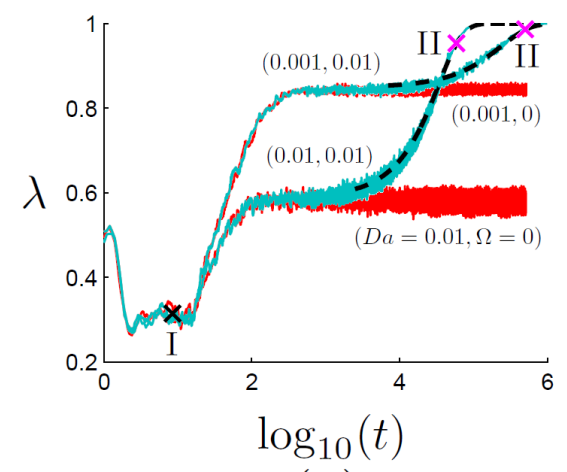

(a)

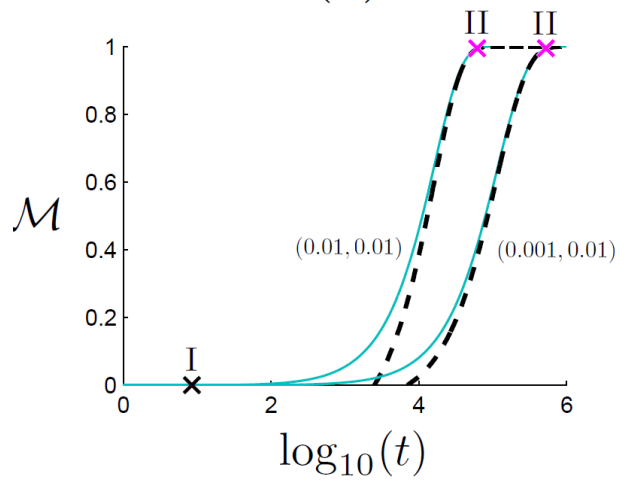

(c)

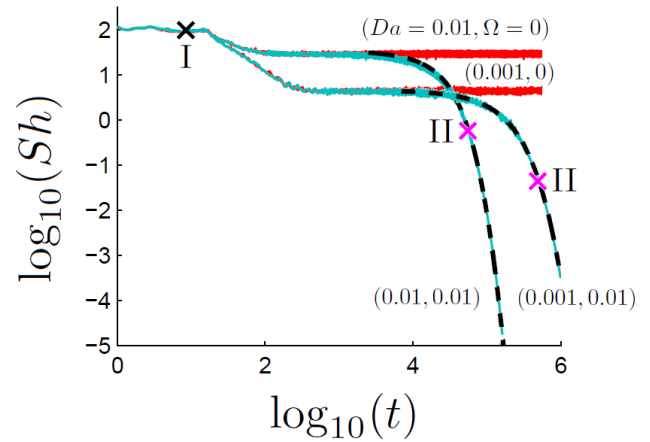

(b)

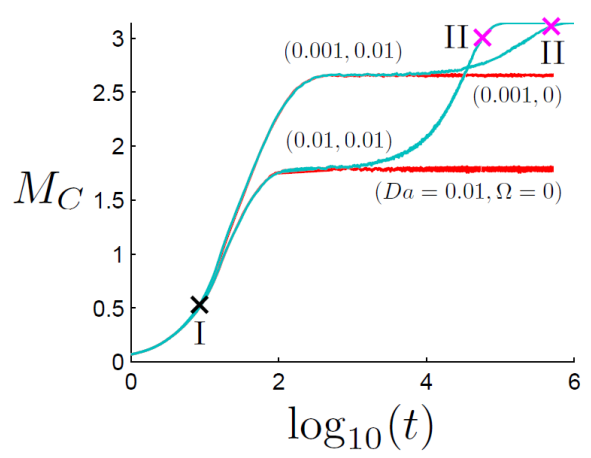

(d)

FIG. 11. Numerical solutions for $R a=5000, L=\pi$ and $(D a, \Omega)=(0.01,0.01)$ and $(0.001,0.01)$ (cyan curves) and $(D a, \Omega)=(0.01,0)$ and $(0.001,0)$ (red curves) calculated from $(4,5) .(a-d)$

respectively show $\lambda, S h, \mathcal{M}$ and $M_{C}$; dashed curves show approximations obtained from $(38,39)$, $J_{0}=D a \lambda \bar{S}$ and $\bar{S}=1$ at $\tau=0$ with $\Delta_{c}=16$ and $\tau_{0}=0.25$ for $D a=0.01$ and $\tau_{0}=0.07$ for $D a=0.001$. Crosses labelled I and II signify the appropriate times at which tips of the plumes first encounter the bottom of the domain and when $\mathcal{M}>0.99$.

a substantial increase in the time from the plumes first encountering the base of the domain (labelled I in Figure 11) to total depletion of $S$ (II).

\section{The effect of changing $\Omega$}

We saw in Figure 7 how, when the initial dimensional concentration of substrate is much smaller than the dimensional concentration of solute at the upper boundary (so that $\Omega \gg 1$ ), 
depletion of $S$ is rapid relative to the timescale for convection and the reaction is limited to an elongated interface between the solute and the unreacted substrate. In contrast, the reaction zone can occupy the full domain when the substrate is abundant relative to the solution $(\Omega \ll 1$, Figure 10$)$. We conclude this section by exploring the effect of changing $\Omega$ on the overall dynamics.

Simulations at different values of $\Omega$ with $D a=1$ show that the penetration depth of the plumes increases approximately linearly in time (Figure 12(a)). The data are plotted to illustrate a scaling relationship for large $\Omega$ derived and discussed in Section IV C below. Increasing $\Omega$ shortens the time for plumes to penetrate to the bottom of the domain, although there is little change in this time for $\Omega$ greater than about 25 (Figure 12(b)). The measure of reacted substrate $\mathcal{M}$ increases linearly and then saturates at unity (Figure $12(d)$ ): the data for $\mathcal{M}$ in the initial phase collapse when scaled by $\Omega /(2+\Omega \lambda)$ with $\lambda=0.29$, as explained below. For $\Omega \gg 1$, the wavenumber is independent of $\Omega$ (Figure 12(c)) and again decays with time.

\section{REDUCED MODELS FOR $R a \gg 1$}

We now extend our previous analysis ${ }^{20}$ of the single-species problem $(\Omega=0)$ to provide approximations of the flow dynamics at high $R a$ when the substrate degrades slowly $(0<$ $\Omega \ll 1)$, rapidly $(\Omega \gg 1)$ and when the overall reaction is slow compared to convective timescales $(D a \ll 1)$. We focus on the structure of the slender plumes beneath the boundary layer, representing the thin dynamic boundary layer near $z=0$ in terms of parameters $J_{0}$ and $\lambda$ that we extract from simulations. We exploit the observation that, as long as the plumes are shorter than the depth of the domain, the dynamic boundary layer adjusts to maintain $J_{0}$ and $\lambda$ at approximately constant values, so that the effects of chemical reaction are felt only within the plumes beneath. While the models are not fully predictive, they provide useful approximations that summarise in mechanistic terms the results of computationally demanding simulations. 


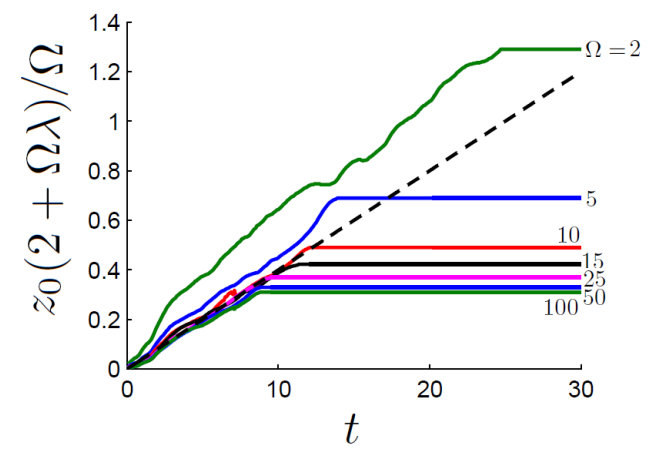

(a)

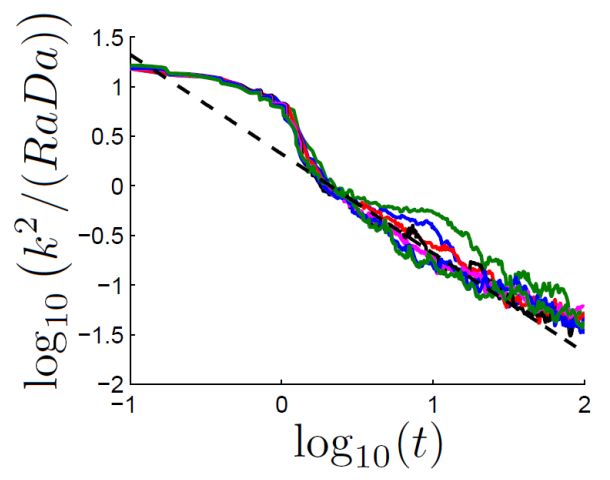

(c)

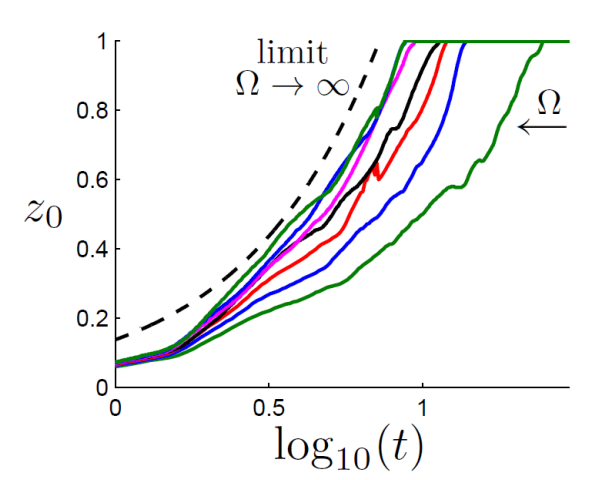

(b)

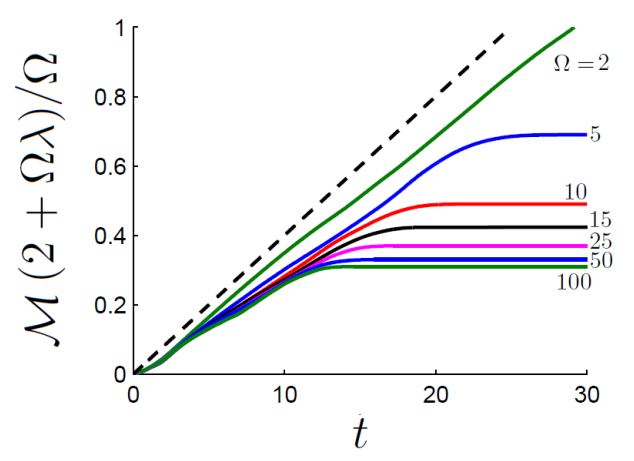

(d)

FIG. 12. (a) Scaled $z_{0}$ calculated from $(4,5)$ using $R a=5000, L=\pi, D a=1, \Omega=0.5,2,5,10,15,25$ and 50. The dashed line shows the large- $\Omega$ approximation (48), for $J_{0}=0.02, \lambda=0.29$. (b) shows $z_{0}$ approaching the large $\Omega$ approximation $2 J_{0} / \lambda$ (dashed line). $(c)$ and $(d)$ show the wavenumbers and amount of depleted substrate for the given $\Omega$; dashed curves show the approximation (48a) and $\mathcal{M}=z_{0}$ using $(48 b)$.

\section{A. A simplified model for slow depletion, $\Omega \ll 1$}

We first assume that $\Omega \ll 1 \ll R a$ with $D a=O(1)$, so that the substrate is sufficiently abundant for its concentration to change relatively slowly compared to the timescale of convective mixing. We focus first on the time during which plumes descend but are yet to 
interact with the bottom of the domain. We assume solutions to $(4,5)$ are of the form

$$
\begin{gathered}
C(x, z, t)=\bar{C}(z, \tau)+C^{\prime}(x, z, t), \quad \psi(x, z, t)=\psi^{\prime}(x, z, t), \\
S=\bar{S}(z, \tau)+S^{\prime}(x, z, t), \quad \tau=\operatorname{Da} \Omega t
\end{gathered}
$$

where $\bar{C}$, and $\bar{S}$ are ensemble averages evolving over the slow timescale $\tau$, and $\overline{C^{\prime}}=\overline{\psi^{\prime}}=$ $\overline{S^{\prime}} \equiv 0$. Here we assume that convective motion over timescales $t=O(1)$, involving plumes that fluctuate laterally, leads to mixing that yields the proposed decomposition, i.e. the ensemble average acts as both a horizontal average and an average over the fast convective timescale $t$. Furthermore, when $\Omega \ll 1$, simulations indicate that this mixing suppresses horizontal concentration gradients in the $S$ field (compare Figures 4(b) and (e)), although convective motions maintain strong horizontal gradients in the $C$ field. Since the plumes are slender structures, we neglect vertical diffusion in comparison to horizontal diffusion, so that (7) reduces to $\psi_{x x}^{\prime}=-C_{x}^{\prime}$. Assuming zero mean vertical flow, it follows that

$$
\psi_{x}^{\prime}=-C^{\prime}
$$

and $(4 b, c)$ becomes

$$
\begin{aligned}
\operatorname{Da} \Omega \bar{C}_{\tau}+C_{t}^{\prime}+\psi_{z}^{\prime} C_{x}^{\prime}+C^{\prime}\left(\bar{C}_{z}+C_{z}^{\prime}\right) & =R a^{-1} C_{x x}^{\prime}-D a\left(\bar{C}+C^{\prime}\right)\left(\bar{S}+S^{\prime}\right), \\
D a \Omega \bar{S}_{\tau}+S_{t}^{\prime} & =-D a \Omega\left(\bar{C}+C^{\prime}\right)\left(\bar{S}+S^{\prime}\right) .
\end{aligned}
$$

Taking the ensemble average of (19) gives

$$
\begin{aligned}
\operatorname{Da\Omega } \bar{C}_{\tau}+\overline{\psi_{z}^{\prime} C_{x}^{\prime}}+\overline{C^{\prime} C_{z}^{\prime}} & =-D a\left(\bar{C} \bar{S}+\overline{C^{\prime} S^{\prime}}\right), \\
\bar{S}_{\tau} & =-\left(\bar{C} \bar{S}+\overline{C^{\prime} S^{\prime}}\right),
\end{aligned}
$$

and the difference between (19) and (20) gives

$C_{t}^{\prime}+\left(\psi_{z}^{\prime} C_{x}^{\prime}-\overline{\psi_{z}^{\prime} C_{x}^{\prime}}\right)+C^{\prime} \overline{C_{z}}+\left(C^{\prime} C_{z}^{\prime}-\overline{C^{\prime} C_{z}^{\prime}}\right)=R a^{-1} C_{x x}^{\prime}-D a\left(\bar{C} S^{\prime}+C^{\prime} \bar{S}+C^{\prime} S^{\prime}-\overline{C^{\prime} S^{\prime}}\right)$,

$$
S_{t}^{\prime}=-D a \Omega\left(\bar{C} S^{\prime}+C^{\prime} \bar{S}+C^{\prime} S^{\prime}-\overline{C^{\prime} S^{\prime}}\right) .
$$


We can scale out $R a$ and $D a$ by defining the new variables $z=\hat{z} / D a, x=\hat{x} /(R a D a)^{1 / 2}$, $\psi^{\prime}=\hat{\psi}^{\prime} /(R a D a)^{1 / 2}$ and $t=\hat{t} / D a$, so that (18), (20) and (21) become

$$
\begin{aligned}
\hat{\psi}_{\hat{x}}^{\prime} & =-C^{\prime} \\
\Omega \bar{C}_{\tau}+\overline{\hat{\psi}_{\hat{z}}^{\prime} C_{\hat{x}}^{\prime}}+\overline{C^{\prime} C_{\hat{z}}^{\prime}} & =-\left(\bar{C} \bar{S}+\overline{C^{\prime} S^{\prime}}\right) \\
\bar{S}_{\tau} & =-\left(\bar{C} \bar{S}+\overline{C^{\prime} S^{\prime}}\right) \\
C_{\hat{t}}^{\prime}+\left(\hat{\psi}_{\hat{z}}^{\prime} C_{\hat{x}}^{\prime}-\overline{\hat{\psi}_{\hat{z}}^{\prime} C_{\hat{x}}^{\prime}}\right)+C^{\prime} \overline{C_{\hat{z}}}+\left(C^{\prime} C_{\hat{z}}^{\prime}-\overline{C^{\prime} C_{\hat{z}}^{\prime}}\right) & =C_{\hat{x} \hat{x}}^{\prime}-\left(\bar{C} S^{\prime}+C^{\prime} \bar{S}+C^{\prime} S^{\prime}-\overline{C^{\prime} S^{\prime}}\right), \\
S_{\hat{t}}^{\prime} & =-\Omega\left(\bar{C} S^{\prime}+C^{\prime} \bar{S}+C^{\prime} S^{\prime}-\overline{C^{\prime} S^{\prime}}\right)
\end{aligned}
$$

The vertical flux then satisfies $J=-\psi_{x} C-C_{z}=-\hat{\psi}_{\hat{x}}^{\prime} C^{\prime}+O\left(R a^{-1}\right)$. In the mass balance equation (10), we expect $M_{C t}$ to vanish (assuming the convection is in a statistically quasisteady state) so that the dominant balance is between the flux of $C$ into the domain and depletion of the substrate. Writing $M=\int_{0}^{D a}(1-\bar{S}) \mathrm{d} \hat{z}$ (measuring deviations from the initial uniform state $\bar{S}=1$ ) and $S h=J_{0} R a$ (representing the flux delivered by the boundary layer at $z=0$, see Figure $5(b)$ and (13)) the mass balance becomes

$$
M_{\tau}=J_{0}
$$

Equivalently, $D a \mathcal{M}_{\tau}=J_{0}$ : we use Figure $9(a)$ to estimate $J_{0} \approx 0.02$ when $R a=5000$, slightly in excess of the expected asymptotic value 0.017 for $R a \rightarrow \infty$.

Equation (22e) implies that perturbations $S^{\prime}$ are weak when $\Omega \ll 1$. Setting $S^{\prime}=\Omega \hat{S}^{\prime}$ and neglecting terms of $O(\Omega),(22)$ becomes

$$
\begin{aligned}
\hat{\psi}_{\hat{x}}^{\prime} & =-C^{\prime}, \\
\overline{\hat{\psi}_{\hat{z}}^{\prime} C_{\hat{x}}^{\prime}}+\overline{C^{\prime} C_{\hat{z}}^{\prime}} & =-\bar{C} \bar{S}, \\
\overline{S_{\tau}} & =-\bar{C} \bar{S}, \\
C_{\hat{t}}^{\prime}+\left(\hat{\psi}_{\hat{z}}^{\prime} C_{\hat{x}}^{\prime}-\overline{\hat{\psi}_{\hat{z}}^{\prime} C_{\hat{x}}^{\prime}}\right)+C^{\prime} \overline{C_{\hat{z}}}+\left(C^{\prime} C_{\hat{z}}^{\prime}-\overline{C^{\prime} C_{\hat{z}}^{\prime}}\right) & =C_{\hat{x} \hat{x}}^{\prime}-C^{\prime} \bar{S},
\end{aligned}
$$

and (22e) decouples from (24). By losing the time derivative in (24b), we lose a description of the transient development of the $\bar{C}$ field. We seek solutions of (24) on the domain $0<$ 
$\hat{z}<\hat{z}_{0}(\tau)$, imposing the boundary conditions

$$
\begin{gathered}
\bar{C}=\lambda, \quad-\overline{\hat{\psi}_{\hat{x}}^{\prime} C^{\prime}}=J_{0} \quad \text { at } \hat{z}=0, \\
\bar{C}=0, \quad \bar{S}=1 \quad \text { at } \hat{z} \rightarrow \hat{z}_{0}(\tau)-, \\
\bar{S}=1 \quad \text { for } \tau=0 .
\end{gathered}
$$

We seek the depth of the plume $\hat{z}_{0}(\tau)$ as a function of the parameters $\lambda$ and $J_{0}$.

Setting

$$
\hat{\psi}^{\prime}=f(\hat{z}, \hat{t}, \tau) \cos \left(\hat{k}\left(\hat{x}-\hat{x}_{0}\right)\right), \quad C^{\prime}=g(\hat{z}, \hat{t}, \tau) \sin \left(\hat{k}\left(\hat{x}-\hat{x}_{0}\right)\right)
$$

for some $\hat{x}_{0}(\tau)$ and $\hat{k}(\tau)$, and substituting into (24) gives

$$
\begin{gathered}
\hat{k} f=g, \quad g g_{\hat{z}}=-\bar{C} \bar{S}, \quad \bar{S}_{\tau}=-\bar{C} \bar{S} \\
g_{\hat{t}}=-g\left(\bar{C}_{\hat{z}}+\hat{k}^{2}+\bar{S}\right) .
\end{gathered}
$$

For $g$ to be as large as possible without growing exponentially, we assume $\bar{C}_{\hat{z}}+\hat{k}^{2}+\bar{S}=0$, where $g \neq 0$, so that $g_{\hat{t}}=0$ and $f_{\hat{t}}=0$. With (24), (25) and (27), we obtain

$$
\begin{gathered}
\bar{C}=\hat{k}^{2}\left(\hat{z}_{0}-\hat{z}\right)+\int_{\hat{z}}^{\hat{z}_{0}} \bar{S} \mathrm{~d} \hat{z}^{\prime}, \quad \lambda=\hat{k}^{2} \hat{z}_{0}+\int_{0}^{\hat{z}_{0}} \bar{S} \mathrm{~d} \hat{z}^{\prime}, \\
\bar{S}_{\tau}=-\bar{S}\left(\hat{k}^{2}\left(\hat{z}_{0}-\hat{z}\right)+\int_{\hat{z}}^{\hat{z}_{0}} \bar{S} \mathrm{~d} \hat{z}^{\prime}\right), \\
\frac{1}{2} g^{2}=\int_{\hat{z}}^{\hat{z}_{0}} \bar{C} \bar{S} \mathrm{~d} \hat{z}^{\prime}, \quad J_{0}=\int_{0}^{\hat{z}_{0}} \bar{C} \bar{S} \mathrm{~d} \hat{z}^{\prime} .
\end{gathered}
$$

Taking $\bar{S}=1$ in $\hat{z}>\hat{z}_{0}$, to leading order the amount of depleted substrate per unit width reduces to

$$
M=\int_{0}^{\hat{z}_{0}}(1-\bar{S}) \mathrm{d} \hat{z}^{\prime}
$$

Differentiating with respect to $\tau$, and using (28a) with the boundary conditions $\bar{S}\left(\hat{z}_{0}, \tau\right)=1$, $g\left(\hat{z}_{0}, \hat{t}, \tau\right)=0$, we recover $(23)$ as

$$
M_{\tau}=\left.\frac{1}{2} g^{2}\right|_{\hat{z}=0} .
$$

Assuming $J_{0}$ is constant and that $M=0$ at $\tau=0,(28 c)$ and (30) give

$$
D a \mathcal{M}=\int_{0}^{\hat{z}_{0}}(1-\bar{S}) \mathrm{d} \hat{z}^{\prime}=J_{0} \tau
$$


Combining (28a) and (31) and setting $\hat{z}=\zeta \hat{z}_{0}(\tau)$, the nonlinear free-boundary problem (28) is mapped onto the fixed domain $0<\zeta<1$ as

$$
\begin{aligned}
\frac{\bar{C}_{\zeta}}{\hat{z}_{0}}+\hat{k}^{2}+\bar{S} & =0, \\
\bar{S}_{\tau}-\zeta \frac{\hat{z}_{0 \tau}}{\hat{z}_{0}} \bar{S}_{\zeta}+\bar{C} \bar{S} & =0, \\
\hat{z}_{0}\left(1-\int_{0}^{1} \bar{S} \mathrm{~d} \zeta\right) & =J_{0} \tau, \\
\hat{z}_{0} & =\frac{\lambda+J_{0} \tau}{1+\hat{k}^{2}},
\end{aligned}
$$

with

$$
\bar{C}(1, \tau)=0, \quad \bar{S}(1, \tau)=1, \quad \bar{S}(\zeta, 0)=1
$$

Because $\lambda$ and $J_{0}$ take prescribed values, $(32,33)$ is parameter-free, consistent with the collapse of data in Figure $9(b)$.

At $\zeta=0, \bar{C}=\lambda$ and $(32 b)$ gives $\bar{S}_{\tau}=-\lambda \bar{S}$, implying that the substrate decays exponentially near the upper boundary. As $\zeta \rightarrow 1-$, expanding $\bar{S}$ as power series in $(1-\zeta)$ in $(32 a, b)$ gives

$$
\bar{C} \approx \hat{z}_{0}\left(1+\hat{k}^{2}\right)(1-\zeta)+\ldots, \quad \bar{S} \approx 1-\hat{z}_{0}\left(1+\hat{k}^{2}\right)(1-\zeta)^{2} /\left(2 \hat{z}_{0 \tau}\right)+\ldots
$$

Because this approximation neglects vertical diffusion, the piecewise linear distribution of $\bar{C}$ will be smoothed by neglected diffusive effects. However (34) explains the quadratic leading-edge structure of $\langle S\rangle$ evident in Figure 3(c), for example.

We solved $(32,33)$ using a numerical scheme outlined in Appendix A. This was validated by comparison with early- and late-time asymptotic approximations, described respectively in Appendices B and C. In particular, we find that

$$
\begin{array}{lll}
z_{0}=\frac{2 J_{0}}{D a \lambda}, \quad k^{2}=\operatorname{RaDa}\left(\frac{\lambda^{2}}{2 J_{0}}-1\right) & \text { for } D a \Omega t \ll 1, \\
z_{0}=J_{0} \Omega t, \quad k^{2}=\frac{\lambda R a}{J_{0} \Omega t} & \text { for } D a \Omega t \gg 1 .
\end{array}
$$

The early-time solution exhibits a weak singularity that propagates from the plumes' leading edge into the main flow domain (Figure 13 below); the late-time solution exhibits a travellingwave structure, with reaction confined to a zone at the plumes' leading edge (Figure 14 below). For plumes to be slender we need $k^{2} z_{0}^{2} \gg 1$, which implies $R a \gg D a$ at early times 
and $R a \Omega t \gg 1$ at late times. In addition, the approximation fails when the plumes first encounter the base of the domain, requiring $t<1 / J_{0} \Omega$.

Solutions of $(32,33)$, based on numerical estimates of $J_{0}=0.02$ (see Figures $5(b)$ and $9(a)$ ) and $\lambda=0.29$ (Figure $5(c)$ ), yield predictions of the plume wavenumber and penetration depth shown in Figures $5(d), 5(a)$ and $9(b)$. The simplified model captures the approximately linear growth of $z_{0}$ and the decay of wavenumber, which takes the simple form (35b) at large times, independent of $D a$. The vertical profiles of horizontally-averaged concentration fields and streamfunction in Figure 4 show good agreement between predictions of the approximate model with solutions to $(4,5)$, outside the boundary layer at $z=0$. Initially (with $\tau \ll 1), \bar{S}$ is approximately linear in $\zeta$, except for a region of width $O(\tau)$ close to $\zeta=1$ (see Appendix B and Figure 13), where the solution adjusts to the quadratic form in (34b). At larger times the agreement with simulation data is not as good, possibly due to the increased $x$-variation of the $S$ field seen in Figure 4(e), or possibly because the solution encounters the base of the domain. However, for $D a \gg 1$ there is sufficient time for the $\bar{S}$-field to adopt a travellingwave form illustrated by Figure 14 below, for which $\bar{C} \rightarrow\left(2 J_{0}\right)^{1 / 2} \approx 0.2$ at the rear of a thin reaction zone. In comparison to the plume length $J_{0} \Omega t$, the reaction is confined to a region of width $D a^{-1}$ at the plume base. (The plumes within the reaction zone remain thin before reaching the base of the domain provided $\left.D a \ll R a^{1 / 2}\right)$.

The strength of perturbations to the mean $C$ field and the streamfunction, plotted in Figure $4(c, d)$, are also captured well by the reduced model. The streamlines extend into $\zeta>1$, representing disturbances that are not captured by the approximate model.

\section{B. A simplified model for slow reaction, $D a \ll 1$}

We now extend the analysis in the previous section to describe features of the flow when $D a \ll 1$. In this case, we replace $(25 b)$ with a no-flux condition $g^{2}=0$ at $z_{0}=1$ (i.e. $\left.\hat{z}_{0}=D a\right)$, requiring us to solve for $\bar{S}$ and $\bar{C}$ over what is effectively a very short domain. Figure 10 shows that changes in $C$ and $S$ are weak across the domain, so that we can assume $\bar{C} \approx \lambda$ to leading order (which varies slowly in time, Figure 11). Equations $(27 a, b)$ then become

$$
\bar{C}=\lambda, \quad \frac{1}{2}\left(g^{2}\right)_{\hat{z}}=-\lambda \bar{S}, \quad \bar{S}_{\tau}=-\lambda \bar{S},
$$


with $\frac{1}{2} g^{2}=J_{0}$ on $\hat{z}=0, g=0$ on $\hat{z}=D a$ and $\bar{S}=1$ when $\tau=0$. Assuming $\bar{S}$ is uniform over $\hat{z},(36 b)$ becomes

$$
\frac{1}{2} g^{2}=J_{0}-\lambda \bar{S} \hat{z}
$$

which implies that $J_{0}=\lambda \bar{S} D a$, and combining this with (13) gives

$$
\bar{S}=\frac{2(1-\lambda)^{2}}{\operatorname{Da\pi } \Delta_{c} \lambda} .
$$

Substituting (38) into (36c) then yields

$$
\lambda_{\tau}=\lambda^{2}\left(\frac{1-\lambda}{1+\lambda}\right)
$$

with initial condition $\bar{S}=1$ in (38), implying $\lambda \rightarrow 1$ as $\tau \rightarrow \infty$. Integrating (39) with respect to $\tau$ gives $\lambda$ with a constant of integration corresponding to a shift in time, $\tau_{0}$.

Solutions of $(38,39)$ using $\Delta_{c}=16$ and $\tau_{0}=0.25$ for $D a=0.01$ and $\tau_{0}=0.07$ for $D a=0.001$ give predictions of $\lambda, S h$ and $\mathcal{M}$ (illustrated in Figure 11). The simplified model captures remarkably well the increase in $\lambda$ and $\mathcal{M}$ and the decay of $S h$ at late times. Figure 10 compares the horizontally-averaged $C$ and $S$ fields for $D a=0.001, \Omega=0.01$ obtained from $(4,5)$ (solid curves) with solutions of $(38,39)$ (dotted curves), capturing the increase in $C$ and decay of $S$ with respect to $t$ at late times.

\section{A simplified model for rapid degradation, $\Omega \gg 1$}

We now develop a more ad hoc, but nevertheless useful, model describing the rapid depletion of $S$ in the limit $R a \gg 1, \Omega \gg 1$ with $D a=O(1)$. In this limit, the substrate is initially present in low concentrations relative to the solute and is immediately degraded as it encounters solute. As illustrated in Figure 7, the $S$ field partitions into distinct chemically inert regions either side of a thin reactive region. While this interface remains continuous as the plumes descend, it is highly irregular, and $\tilde{S}$ is no longer small compared to the horizontal average (Figure 8e). Even so, we find that a Reynolds decomposition such as (17) still yields valuable predictions. The calculation that follows lacks the rigour of Sections IV A, B and should be interpreted more as a scaling argument than a rational asymptotic derivation, although the resulting predictions are surprisingly accurate.

First, it is helpful to recall the specialised case in which the interface is uniform at $z=z_{0}(t)$ and that, as the interface advances slowly into unreacted substrate, there is a dominant 
balance between reaction and diffusion, with $C=0$ and $S=1$ ahead of $z=z_{0}$ (a region denoted "+") and $C>0, S=0$ behind ("-"). The intermediate reactive region is governed by

$$
-z_{0 t} C_{z}=R a^{-1} C_{z z}-D a C S, \quad-z_{0 t} S_{z}=-D a \Omega C S
$$

Integrating across the thin reactive zone in which $C S>0$, assuming that $S$ enters the front $(+)$ of the reactive zone with advective flux $z_{0 t}$, while $C$ enters the rear $(-)$ with diffusive flux $-R a^{-1} C_{z}$ (at a much greater rate than it is advected out), the solute flux is ${ }^{12}$

$$
-\left.R a^{-1} C_{z}\right|_{-}=D a \int_{-}^{+} C S \mathrm{~d} z=z_{0 t} / \Omega
$$

We now return to the slender plume approximation, ${ }^{20}$ assuming $S$ is completely depleted in the bulk of the plumes (in $0<z<z_{0}(t)$ ) but ensuring that the plumes deliver a solute flux of magnitude $z_{0 t} / \Omega$ at the base, consistent with (41). (We do not match the plume region directly to the thin reaction zone, but rely instead on the requirement that fluxes must balance over an intermediate boundary layer.) As before, we assume the boundary layer at $z=0$ maintains a fixed solute flux $J_{0}$ and subsurface average concentration $\lambda$. Then, in the plume region, we consider $(4,5)$ without the reaction and vertical diffusion terms, and set

$$
C(x, z, t)=\bar{C}(z, t)+g(z, t) \sin (k x), \quad \psi(x, z, t)=f(z, t) \cos (k x)
$$

where the bar now denotes a horizontal average. The solute transport and Darcy equations give respectively

$$
\left(\bar{C}_{t}+g g_{z}\right)+\left(g_{t}+g \bar{C}_{z}+g R a^{-1} k^{2}\right) \sin (k x)=0, \quad g=f k,
$$

which are satisfied by

$$
\bar{C}_{t}+g g_{z}=0, \quad g_{t}+g\left(\bar{C}_{z}+k^{2} / R a\right)=0 .
$$

For $g$ to be neutrally stable (44) gives

$$
\bar{C}=\lambda-R a^{-1} k^{2} z
$$

where $\bar{C}(0)=\lambda$ is constant. The vertical flux satisfies $J=-\psi_{x} C+O\left(R a^{-1}\right)$ and so $\left.\frac{1}{2} g^{2}\right|_{z=0}=J_{0}$. Equation (44) then also gives

$$
\frac{1}{2} g^{2}=J_{0}+\frac{1}{2} R a^{-1}\left(k^{2}\right)_{t} z^{2} .
$$


(We assume here a slow time-dependence in $k^{2}$, even though it was neglected in deriving (42); its earlier retention would otherwise require an alternative closure approximation.) The dominant vertical solute flux at the plume base is $\frac{1}{2} g^{2}$, so setting $\frac{1}{2} g^{2}=z_{0 t} / \Omega$ and $\bar{C}=0$ at $z=z_{0}-$, gives

$$
J_{0}+\frac{1}{2} R a^{-1}\left(k^{2}\right)_{t} z_{0}^{2}=\Omega^{-1} z_{0 t}, \quad\left(k^{2}\right)_{t}=\operatorname{Ra} \lambda \frac{z_{0 t}}{z_{0}^{2}},
$$

which combine to yield $z_{0 t}=2 J_{0} \Omega /(2+\Omega \lambda)$. Thus at large times we obtain the approximation

$$
k^{2}=\left(\frac{2+\Omega \lambda}{2 J_{0} \Omega}\right) \frac{R a \lambda}{t}, \quad z_{0}=\left(\frac{2 J_{0} \Omega}{2+\Omega \lambda}\right) t .
$$

For $\Omega \gg 1$, (48) reduces to $\Omega$-independent limits $k^{2}=R a \lambda^{2} /\left(2 J_{0} t\right)$ and $z_{0}=2 J_{0} t / \lambda$.

Using again the numerical estimates $J_{0} \approx 0.02$ and $\lambda \approx 0.29$, we compare the predictions (48) of plume wavenumber and penetration depth to simulation data in Figures 5(d) and 12(a) respectively. The reduced model captures the linear growth of $z_{0}$ for $\Omega \geq 5$ well and predicts the minimum time for the plumes to penetrate to the bottom of the domain in the singular limit $\Omega \rightarrow \infty$ (Figure 12(b)). The simplified model also predicts the $t^{-1 / 2}$ decay of the plume wavenumber while the plumes are descending into the domain.

Despite the large horizontal gradients in the concentration fields (Figures 6, 7), the approximate model is surprisingly successful in capturing horizontally-averaged profiles of $C$, root mean square $\tilde{C}$ and $\psi$ (Figure 8 ). Equation (45) predicts that $\langle C\rangle$ decays linearly with $z$, a feature evident in simulation data. Naturally the plume model predicts a sharp interface in $\bar{S}$ between the plumes and unreacted solute; this feature is not reflected in Figure $8(b)$ because of the strong variation of individual plume penetration depths. The strength of perturbations to the mean $C$-field and the stream function (Figures $8(c, d)$ ) are also well captured by the simplified model. Numerical results show that the stream function senses the effect of the lower boundary before the plumes penetrate to the base of the domain; this may explain the deviation in $\tilde{C}$ and $\psi$ obtained numerically and from the reduced model at later times.

\section{DISCUSSION}

This study of high-Rayleigh-number porous-medium convection has demonstrated the pivotal role of the thin sub-source boundary layer in regulating the flux of solute into the 
domain. By comparing simulations with lower-order models, we have provided evidence that the boundary layer is not directly affected by the nature and rate of chemical reactions elsewhere in the domain. This observation underpins our upscaling approximations for the convective plumes that penetrate deep into the flow domain. These exploit the horizontal mixing induced by the fluctuating plumes, enabling us to describe the vertical structure of the flow field in terms of horizontal averages.

Our previous study ${ }^{20}$ described statistically-steady convection across a range of $D a$ for $R a \gg 1$, equivalent to the singular limit $\Omega=0$ of the present problem. Convection arises for $R a^{-2} \ll D a \ll R a$ : large $D a$ (rapid reaction) leads to convection being restricted to a region near the top of the domain; small $D a$ leads to solute filling the full depth of the domain. In the present case, with a finite amount of substrate in the system $(\Omega>0)$, the convection is guaranteed to shut down at sufficiently large times. Integrating (10) from the initial state $(C, S)=(0,1)$ to the final state $(1,0)$ shows that the amount of solute absorbed per unit length is proportional to $1+(1 / \Omega)$; the second term represents the component which reacts with the substrate. It is less obvious how rapidly the solute is taken up. We therefore distinguish key events in the dynamics: (I) the moment when plumes first meet the base of the domain; (II) the moment when the substrate is eliminated; and the later time when the system finally reaches equilibrium. Approximations of such quantities can give useful physical estimates of the long-term storage capacity of potential storage sites. Given the uncertainties and variabilities of the parameter values arising in specific applications, however ( $D a$ is estimated to lie in the range $10^{-2}$ to $10^{5}$, for example ${ }^{10}$ while $\Omega$ was estimated above to be $O(1)$ for the Sleipner field but is likely to be an order of magnitude larger or smaller at other sites), it is helpful to survey the full parameter space; our limiting approximations for large and small values of $\Omega$ (e.g. Figures 5 and 12) help bound likely outcomes.

The parameter $\Omega$ measures the relative amounts of solute and substrate. Small $\Omega$ corresponds to abundant substrate, which consequently takes a long-time to degrade completely. For $D a$ of order unity we have derived a novel nonlinear free-boundary problem $(32,33)$ describing the slow erosion of the substrate, formulated as an integro-differential equation (in one space coordinate and time) coupled to algebraic conditions. Figure 4 shows that the reduced model agrees well with simulations. For $1 \ll D a \ll R a^{1 / 2}$ with $\Omega \ll 1$, the flow initially resembles the $\Omega=0$ limit, but $S$ then starts to decay over a slow timescale. 
Eventually a travelling wave structure emerges in the $S$ field, with a reaction zone (of thickness $O(1 / D a))$ propagating steadily downwards into the domain, at the base of plumes of length $O(\Omega t)$; the plumes therefore meet the base of the domain in a time of $O(1 / \Omega)$ (on the convective timescale). Since the substrate is eliminated behind the the travelling wave, the system enters a shut-down phase promptly thereafter (Figure $5, \Omega=0.01$ ). The slow depletion of the substrate allows the plumes to elongate substantially in comparison to the $\Omega=0$ case. The reaction zone at the base of the plumes (Figure 14) differs from the usual thin zone arising in pure reaction-diffusion systems (with a balance between vertical diffusion and reaction); here, convection and horizontal diffusion play a central role.

When both $D a$ and $\Omega$ are small, a further sequence of events emerges (Figure 11). First, because $D a$ is small, the plumes initially grow downwards into the domain in a manner characteristic of traditional one-sided porous-medium convection, ${ }^{1}$ at a rate regulated by the sub-surface boundary layer. Once they encounter the base of the domain, there is then a box-filling adjustment of the whole flow until there is an approximate balance between the flux through the boundary layer and reaction with the substrate. The substrate then decays on a long timescale of order $O\left((D a \Omega)^{-1}\right)$. Thus events I and II are widely separated in time (Figure 11).

For large $\Omega$, when the solute is scarce, a more traditional (and thinner) reaction front emerges. Descending plumes erode vertical channels in the substrate and the pattern of the $S$ field is strongly heterogeneous (Figure 7). Nevertheless, an approximation based on horizontal averaging still proves useful (Figure 8). Again, the convection enters a shut-down phase soon after plumes reach the base of the domain (Figure $5, \Omega=25$ ), which takes place in order unity time (on the convective timescale).

Numerous features of real geological formations have been neglected in this study, in particular changes to porosity and permeability of the host matrix as a result of the surface reaction with the solute ${ }^{29,30}$ and transport of the reacting mineral due to the flow. ${ }^{31,32}$ We also neglect heterogeneity and anisotropy of the host material, ${ }^{33,34}$ heterogeneity of the source, ${ }^{35}$ solute dispersion ${ }^{36}$ and three-dimensionality. ${ }^{15,37}$ However we anticipate that many of the physical processes described here will be applicable to flows in more realistic practical applications. 


\section{ACKNOWLEDGEMENT}

We are grateful to Dr C. A. Rochelle and Dr D. Noy of the British Geological Survey for valuable discussions. This project was in part funded by the Nottingham Centre for Carbon Capture and Storage and the EU FP7 PANACEA project 282900.

\section{Appendix A: Numerical solution scheme of reduced model for slow degradation}

Equations $(32,33)$ were solved numerically using a spectral method, approximating $\bar{S}$ as a sum of $N$ rescaled Chebyshev polynomials and solved at each time-step using an iterative

process. $\bar{S}$ was integrated over the domain by evaluating the sum of the product of $\bar{S}$ and the Clenshaw-Curtis weights at each of the $N$ collocation points.

Differentiating $(32 c)$ with respect to $\tau$ gives

$$
\hat{z}_{0 \tau}=\frac{\hat{z}_{0}}{\tau}+\frac{\hat{z}_{0}^{2}}{J_{0} \tau} \int_{0}^{1} \bar{S}_{\tau} \mathrm{d} \zeta
$$

then $(32 b)$ was discretised using the scheme

$$
\frac{\bar{S}^{n+1}-\bar{S}^{n}}{\delta \tau}-\frac{\zeta}{\tau^{n+1}} \mathrm{D}_{\zeta} \bar{S}^{n+1}-\frac{\zeta z_{0}^{\mathrm{I}}}{J_{0} \tau^{n+1}} \mathrm{D}_{\zeta} S^{\mathrm{I}}\left(\frac{\mathrm{W} \bar{S}^{n+1}-\mathrm{W} \bar{S}^{n}}{\delta \tau}\right)+C^{\mathrm{I}} \bar{S}^{n+1}=0,
$$

where $\mathrm{D}_{\zeta}$ and $\delta \tau$ are the first-order differential operator acting on the rescaled Chebyshev polynomials and the time-step. W is a $N \times N$ matrix comprising $N$ rows of the ClenshawCurtis weights. $S^{\mathrm{I}}, C^{\mathrm{I}}$ and $z_{0}^{\mathrm{I}}$ are approximations to $\bar{S}^{n+1}, \bar{C}^{n+1}$ and $\hat{z}_{0}^{n+1}$ respectively. At a given time-step, the solution to (A2) was used as the value for $S^{\mathrm{I}}$, and used with $(32 c, d, a)$ to find $C^{\mathrm{I}}$ and $z_{0}^{\mathrm{I}}$ in the next iteration, and the process continued until the difference between $\bar{S}$ and $S^{\mathrm{I}}$ fell below a given threshold. Equation (A2) is singular at $\tau=0$; the small $\tau$ asymptotic approximation (B2) was used as the initial condition at $\tau=\delta \tau$ (e.g. $\delta \tau=10^{-6}$ ).

\section{Appendix B: Early time asymptotics of $(32,33): \tau \ll 1$}

At small times we distinguish between an outer region where $|1-\zeta|=O(1)$ and an inner region where $\zeta$ is close to 1 . In the outer region we assume expansions of the form

$$
\begin{array}{cl}
\bar{C}=C_{0}+C_{1} \tau+C_{2} \tau^{2}+\ldots, & \bar{S}=1+S_{1} \tau+S_{2} \tau^{2}+\ldots, \\
\hat{z}_{0}=z_{00}+z_{01} \tau+z_{02} \tau^{2}+\ldots, & \hat{k}^{2}=k_{0}^{2}+k_{1}^{2} \tau+k_{2}^{2} \tau^{2}+\ldots
\end{array}
$$


Combining (B1) with (32) produces the expansions

$$
\begin{aligned}
\bar{S} & =1-\lambda(1-\zeta) \tau+\left(z_{01} \frac{\lambda^{2}}{4 J_{0}} \zeta-\frac{J_{0}}{2}(1-\zeta)+\frac{1}{2}(1-\zeta)^{2}\left(J_{0}+\lambda^{2}\right)\right) \tau^{2}+\ldots \\
\bar{C} & =\lambda(1-\zeta)+\left(\frac{2 J_{0}}{\lambda}\left(k_{1}^{2}(1-\zeta)-\frac{\lambda}{2}(1-\zeta)^{2}\right)+z_{01} \frac{\lambda^{2}}{2 J_{0}}(1-\zeta)\right) \tau+\ldots \\
\hat{z}_{0} & =\frac{2 J_{0}}{\lambda}+\frac{4 J_{0}}{3}\left(1-\frac{J_{0}}{2 \lambda^{2}}\right) \tau+O\left(\tau^{2}\right), \\
\hat{k}^{2} & =\left(\frac{\lambda^{2}}{2 J_{0}}-1\right)+\frac{\lambda}{3 J_{0}}\left(2 J_{0}-\lambda^{2}\right) \tau+O\left(\tau^{2}\right) .
\end{aligned}
$$

Taking $\lambda \approx 0.29$ and $J_{0} \approx 0.02$, (B2) implies $\hat{k}^{2}$ initially decays linearly with $\tau$. We know from (34) that $\bar{S}$ should be quadratic in $(1-\zeta)$ as $\zeta \rightarrow 1_{-}$, indicating the existence of an additional region close to $\zeta=1$. The $\bar{S}_{\zeta}$ term in $(32 b)$ is $O(\tau)$ and becomes important when $|1-\zeta|=O(\tau)$.

In the inner region we define the variable $\hat{\zeta}=(1-\zeta) / \tau=O(1)$; the inner form of (B2) becomes

$$
\bar{S}=1+\tau^{2}\left(-\lambda \hat{\zeta}+z_{01} \frac{\lambda^{2}}{4 J_{0}}\right)+\tau^{3}\left(-z_{01} \frac{\lambda^{2}}{4 J_{0}} \hat{\zeta}-\frac{J_{0}}{2} \hat{\zeta}\right)+O\left(\tau^{4}\right) .
$$

Writing (32b) in the inner region variables gives

$$
\tau \bar{S}_{\tau}-\hat{\zeta} \bar{S}_{\hat{\zeta}}-\tau \frac{\hat{z}_{0 \tau}}{\hat{z}_{0}}(1-\hat{\zeta} \tau) \bar{S}_{\hat{\zeta}}=-\hat{z}_{0} \bar{S}\left(\hat{k}^{2} \tau^{2} \hat{\zeta}+\tau^{2} \int_{0}^{\hat{\zeta}} \bar{S} \mathrm{~d} \hat{\zeta}^{\prime}\right)
$$

Assuming expansions of the form $\bar{S}=1-\tau^{2} f_{2}(\hat{\zeta})+\ldots$, (B4) gives at $O(\tau)$

$$
\left(\hat{\zeta}-\frac{z_{01}}{z_{00}}\right) f_{2 \hat{\zeta}}-2 f_{2}=-\lambda \hat{\zeta}
$$

Equation (B5) is singular at $\hat{\zeta}=z_{01} / z_{00}$; using the boundary condition $f_{2}(0)=0$ and ensuring continuity across $\hat{\zeta}=z_{00} / z_{01}$, we find

$$
f_{2}(\hat{\zeta})= \begin{cases}\lambda z_{00} \hat{\zeta}^{2} /\left(2 z_{01}\right), & \hat{\zeta} \leq z_{01} / z_{00} \\ \lambda \hat{\zeta}-\lambda z_{01} /\left(2 z_{00}\right), & \hat{\zeta}>z_{01} / z_{00}\end{cases}
$$

which matches with (B3). The weak singularity emerging in the inner solution must persist at larger times and will move into the central part of the domain.

Figures $13(a, b)$ show a comparison between the solution to (32) (solid curves) and the $\tau \ll 1$ asymptotic expansions (B2a) (dotted curves) and (B3) (dots, plotted in the innerexpansion variable $\hat{\zeta})$ to $O\left(\tau^{2}\right)$ respectively, showing good agreement for $\tau<1$. 


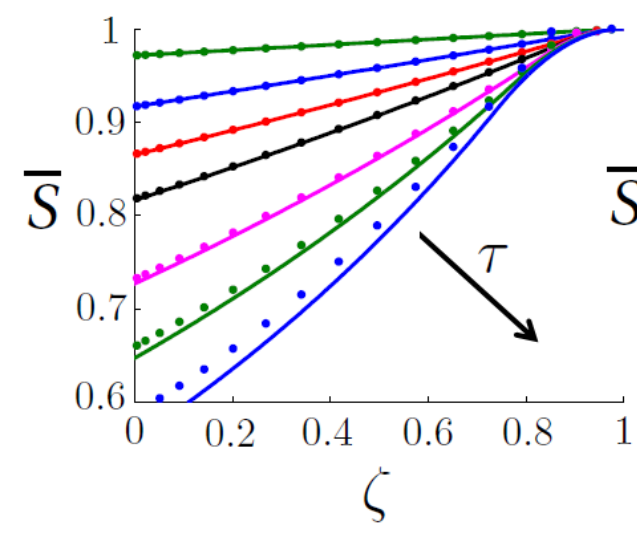

(a)

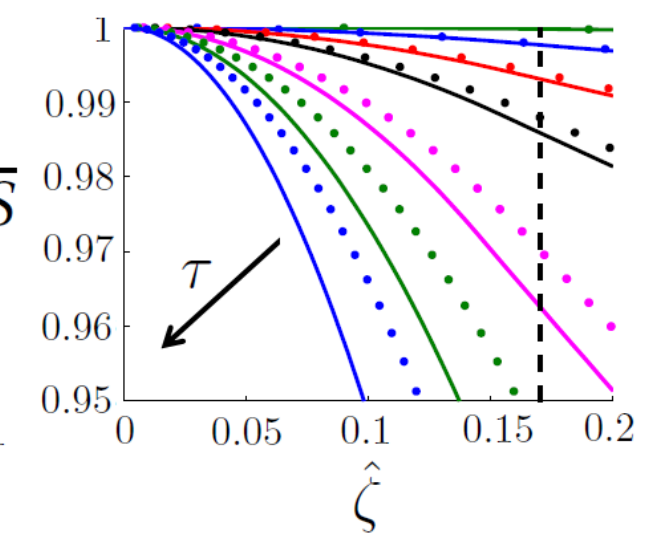

(b)

FIG. 13. (a) Comparison of solutions to (32) (solid curves) and the $\tau \ll 1$ outer approximation (B2) (dots) for $J_{0}=0.02, \lambda=0.29$ at $\tau=0.1,0.3,0.5,0.7,1,1.5$ and 2 . (b) shows the $\tau \ll 1$ inner solution (dots), and the solution to (32) plotted in the inner-region variable $\hat{\zeta}=(1-\zeta) / \tau$. The dashed line in (b) illustrates the location of the singularity in the inner solution at $\hat{\zeta}=z_{01} / z_{00} \approx 0.17$

\section{Appendix C: Late time asymptotics of $(32,33)$ for $D a \gg 1$}

For $\tau \gg 1$ we define $\bar{S}=G(\eta)$ where $\eta=\tau(1-\zeta)$; (32) becomes

$$
\begin{gathered}
\frac{\eta}{\tau} G_{\eta}=-\tau \frac{\hat{z}_{0 \tau}}{\hat{z}_{0}}\left(1-\frac{\eta}{\tau}\right) G_{\eta}-\hat{z}_{0} G\left(\frac{\eta}{\tau} \hat{k}^{2}-\frac{1}{\tau} \int_{\eta}^{0} G\right) \mathrm{d} \eta^{\prime} \\
\hat{z}_{0} \int_{0}^{\tau}(1-G) \mathrm{d} \eta=J_{0} \tau^{2}, \quad \hat{z}_{0}\left(1+\hat{k}^{2}\right)=\lambda+J_{0} \tau
\end{gathered}
$$

and combining $(\mathrm{C} 1 b)$ gives

$$
\hat{k}^{2}=\frac{\lambda}{\hat{z}_{0}}-\frac{1}{\tau} \int_{0}^{\tau} G \mathrm{~d} \eta
$$

To leading order

$$
\hat{z}_{0}=J_{0} \tau, \quad \hat{k}^{2}=\frac{1}{\tau}\left(\frac{\lambda}{J_{0}}-\int_{0}^{\tau} G \mathrm{~d} \eta\right),
$$

and so, to leading order order, $(\mathrm{C} 1 a)$ gives

$$
G_{\eta}+J_{0} G \int_{0}^{\eta} G \mathrm{~d} \eta^{\prime}=0
$$




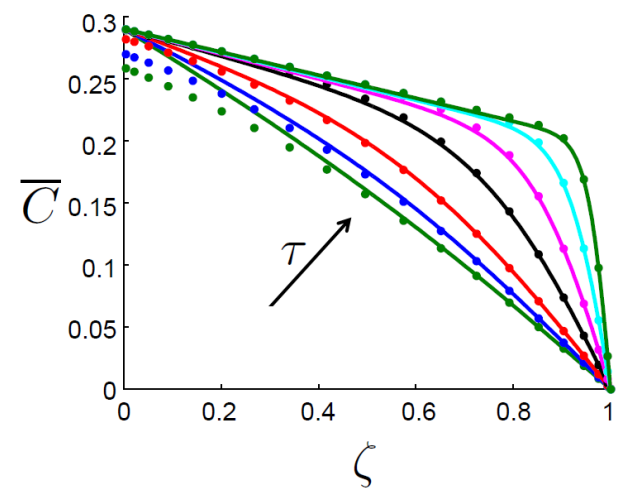

(a)

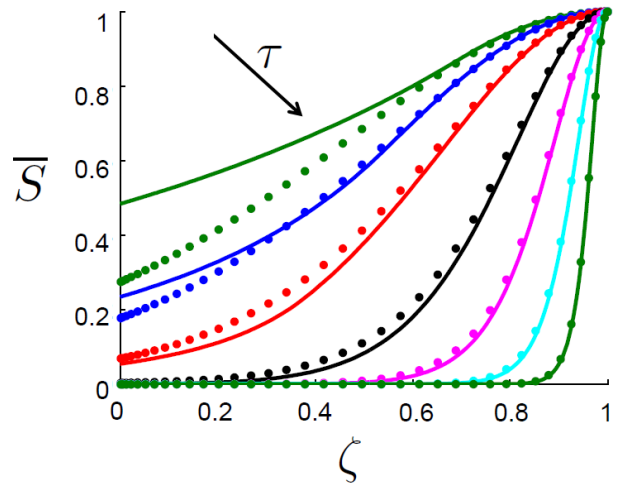

(b)

FIG. 14. Comparison of solution to (32) with $J_{0}=0.02, \lambda=0.29$ (solid) with the solutions to the large-time asymptotic approximation (C5) (dots) plotted at $\tau=5,10,25,50,100$ and 200.

subject to $G=1$ at $\eta=0$ and $G \rightarrow 0$ for $\eta \gg 1$. Writing $G(\eta)=\hat{G}(\hat{\eta})$ where $\eta=\hat{\eta} / J_{0}^{1 / 2}$, (C4) becomes

$$
(\log \hat{G})_{\hat{\eta}}=-\sqrt{2(1-\hat{G})}
$$

so $G_{\hat{\eta}} / G \rightarrow-\sqrt{2}$ as $\hat{\eta} \rightarrow \infty$, implying $\hat{\gamma} \equiv \int_{0}^{\infty} \hat{G}(\hat{\eta}) \mathrm{d} \hat{\eta}=\sqrt{2}$, and for $\eta \gg 1$

$$
G=\exp \left(-J_{0}^{1 / 2} \hat{\gamma} \eta\right) \quad \hat{k}^{2}=\frac{1}{\tau}\left(\frac{\lambda}{J_{0}}-\frac{\hat{\gamma}}{J_{0}^{1 / 2}}\right)
$$

Figure 14 demonstrates how the numerical solution to (32) converges to the asymptotic solution (C5) as $\tau$ increases.

\section{REFERENCES}

${ }^{1}$ A.C. Slim. Solutal-convection regimes in a two-dimensional porous medium. J. Fluid Mech., 741:461-491, 2014.

${ }^{2}$ H.E. Huppert and J.A. Neufeld. The fluid mechanics of carbon dioxide sequestration. Ann. Rev. Fluid Mech., 46:255-272, 2014.

${ }^{3}$ W.D. Gunter, E.H. Perkins, and T.J. McCann. Aquifer disposal of $\mathrm{CO}_{2}$-rich gases: reaction design for added capacity. Proceedings of the IEA carbon dioxide disposal symposium, 34 (Elsevier), 1993. doi:10.1016/0196-8904(93)90040-H. 
${ }^{4}$ W.D. Gunter, B. Wiwchar, and E.H. Perkins. Aquifer disposal of $\mathrm{CO}_{2}-$ rich greenhouse gases: extension of the time scale of experiment for $\mathrm{CO}_{2}$-sequestering reactions by geochemical modelling. Minerology and Petrology, 59:121-140, 1997.

${ }^{5}$ W.D. Gunter, E.H. Perkins, and I. Hutcheon. Aquifer disposal of acid gases: modelling of water-rock reactions for trapping of acid wastes. Applied Geochemistry, 15:1085-1095, 2000.

${ }^{6}$ S.J. Baines and R.H. Worden. Geological $\mathrm{CO}_{2}$ disposal: understanding the long term fate of $\mathrm{CO}_{2}$ in naturally occurring accumulations. Fifth International Conference on Greenhouse Gas Control Technologies (CSIRO, Collingwood), pages 311-316, 2000.

${ }^{7}$ M.N. Watson, N. Zwingmann, N. Lemon, and P.R. Tingate. Onshore Otway Basin carbon dioxide accumulations: $\mathrm{CO}_{2}$-induced diagenesis in natural analogues for underground storage of greenhouse gas. APPEA J, 43(1):183-198, 2003.

${ }^{8}$ C.A. Rochelle, I. Czernichowski-Lauriol, and A.E. Milodowski. The impact of chemical reactions on $\mathrm{CO}_{2}$ storage in geological formations: a brief review. In S.J. Baines and R.H. Worden, editors, Geological Storage of Carbon Dioxide. Geological Society, London, Special Publications 233:87-106, 2004.

${ }^{9}$ M.J. Mitchell, O.E. Jensen, K.A. Cliffe, and M.M. Maroto-Valer. A model of carbon dioxide dissolution and mineral carbonation kinetics. Proc. R. Soc. A, 466:1265-1290, 2010.

${ }^{10} \mathrm{~K}$. Ghesmat, H. Hassanzadeh, and J. Abedi. The impact of geochemistry on convective mixing in a gravitationally unstable diffusive boundary layer in porous media : $\mathrm{CO}_{2}$ storage in saline aquifers. J. Fluid Mech., 673:480-512, 2009.

${ }^{11}$ H. Emami-Meybodi, H. Hassanzadeh, C.P. Green, and J. Ennis-King. Convection dissolution of $\mathrm{CO}_{2}$ in saline aquifers: Progress in modeling and experiments. International Journal of Greenhouse Gas Control, 2015.

${ }^{12}$ J. Ennis-King and L. Paterson. Coupling of geochemical reactions and convective mixing in the long-term geological storage of carbon dioxide. Int. J. Greenhouse Gas Cont., 1: 86-93, 2007.

${ }^{13} \mathrm{~J}$. Ennis-King and L. Paterson. Role of convective mixing in the long-term storage of carbon dioxide in deep saline formations. SPE Journal, 10:349-356, 2005.

${ }^{14}$ A. Riaz, M. Hesse, H.A. Tchelepi, and F.M. Orr. Onset of convection in a gravitationally unstable diffusive boundary layer in porous media. J. Fluid Mech., 548:87-111, 2006. 
${ }^{15}$ G.S.H. Pau, J.B. Bell, K. Pruess, A.S. Almgren, M.J. Lijewski, and K. Zhang. Highresolution simulation and characterization of density-driven flow in $\mathrm{CO}_{2}$ storage in saline aquifers. Adv. Water Res., 33:443-455, 2010.

${ }^{16}$ S. Backhaus, K. Turitsyn, and R.E. Ecke. Convective instability and mass transport of diffusion layers in a Hele-Shaw geometry. Phys. Rev. Lett., 106:104501, 2011.

${ }^{17}$ D.R. Hewitt, J.A. Neufeld, and J.R. Lister. Convective shutdown in a porous medium at high Rayleigh number. J. Fluid Mech., 719:551-586, 2013.

${ }^{18}$ D.R. Hewitt, J.A. Neufeld, and J.R. Lister. Stability of columnar convection in a porous medium. J. Fluid Mech., 737:205-231, 2013.

${ }^{19}$ T.J. Ward, K.A. Cliffe, O.E. Jensen, and H. Power. Dissolution-driven porous-medium convection in the presence of chemical reaction. J. Fluid Mech., 747:316-349, 2014.

${ }^{20}$ T.J. Ward, O.E. Jensen, H. Power, and D.S. Riley. High Rayleigh-number convection of a reactive solute in a porous medium. J. Fluid Mech., 760:95-126, 2014.

${ }^{21}$ J.T.H. Andres and S.S.S. Cardoso. Onset of convection in a porous medium in the presence of chemical reaction. Phys. Rev. E, 83:046312, 2011.

${ }^{22}$ J.T.H. Andres and S.S.S. Cardoso. Convection and reaction in a diffusive boundary layer in a porous medium: Nonlinear dynamics. Chaos, 22(3):037113, 2012.

${ }^{23}$ T. Xu, J.A. Apps, and K. Preuss. Reactive geochemical transport simulation to study mineral trapping for $\mathrm{CO}_{2}$ disposal in deep arenaceous formations. J. Geophys. Res 108, page B2 2071, 2003.

${ }^{24}$ B. Zerai, B.Z. Saylor, and G. Matisof. Computer simulation of $\mathrm{CO}_{2}$ trapped through mineral precipitation in the Rose Run Sandstone, Ohio. Applied Geochemistry, 21:223$240,2006$.

${ }^{25}$ M. Bickle, A. Chadwick, H.E. Huppert, M.A. Hallworth, and S. Lyle. Modelling carbon dioxide accumulation at sleipner: Implications for underground carbon storage. Earth Planetary Sci. Lett., 255:164-176, 2007.

${ }^{26}$ J.M. Pearce, S.J. Kemp, and P.D. Wetton. Mineralogical and petrographical characterisation of a $1 \mathrm{~m}$ core from the Utsira formation, central North Sea. Technical report, 1999. British Geological Survey Report number: WG/99/24C.

${ }^{27}$ L.N. Trefethen. Spectral Methods in MATLAB, volume 10. Society for Industrial and Applied Mathematics, 2000. 
${ }^{28}$ R. Asselin. Frequency filter for time integrations. Monthly Weather Rev., 100(6):487-490, 1972 .

${ }^{29}$ L.T. Ritchie and D. Pritchard. Natural convection and the evolution of a reactive porous medium. J. Fluid Mech., 673:286-317, 2011.

${ }^{30} \mathrm{X} . \mathrm{Fu}, \mathrm{L}$. Cueto-Felgueroso, and R. Juanes. Pattern formation and coarsening dynamics in three-dimensional convective mixing in porous media. Phil. Trans. Roy. Soc. A: Math., Phys. Eng. Sci., 371(2004):20120355, 2013.

${ }^{31} \mathrm{M}$. Kim. Effect of the irreversible $\mathrm{A}+\mathrm{B} \rightarrow \mathrm{C}$ reaction on the onset and the growth of the buoyancy instability in a porous medium. Chemical Engineering Science, 112:56-71, 2014.

${ }^{32}$ C. Almarcha, Y.R. Honi, Y. De Decker, P.M.J. Trevelyan, K. Eckert, and A. De Wit. Convective mixing induced by acid-base reactions. J. Phys. Chem B, pages 9739-9744, 2011.

${ }^{33}$ S. Rapaka, R.J. Pawar, P.H. Stauffer, D. Zhang, and S. Chen. Onset of convection over a transient base-state in anisotropic and layered porous media. J. Fluid Mech., 641:227-244, 2009.

${ }^{34} \mathrm{M}$. Bestehorn and A. Firoozabadi. Effect of fluctuations on the onset of density-driven convection in porous media. Phys. Fluids, 24:114102, 2012.

${ }^{35}$ M.L. Szulczewski, M.A. Hesse, and R. Juanes. Carbon dioxide dissolution in structural and stratigraphic traps. J. Fluid Mech., 736:287-315, 2013.

${ }^{36}$ C. Zhao, B.E. Hobbs, and A. Ord. Theoretical analysis of the effects of solute dispersion on chemical-dissolution front instability in fluid-saturated porous media. Transp Porous Med, 84:629-653, 2010.

${ }^{37}$ D.R. Hewitt, J.A. Neufeld, and J.R. Lister. High Rayleigh number convection in a threedimensional porous medium. J. Fluid Mech., 748:879-895, 2014. 